\title{
Study of Exponential Thermal Boundary Condition on Unsteady Magnetohydrodynamic Convection in a Square Enclosure Filled with $\mathrm{Fe}_{3} \mathrm{O}_{4}$ - Water Ferrofluid
}

\author{
Eare Md. Morshed Alam ${ }^{*}$, M. M. Rahman ${ }^{2}$, Md. Sharif Uddin ${ }^{3}$ \\ ${ }^{1,3}$ Department of Mathematics, Jahangirnagar University, Dhaka, BANGLADESH \\ ${ }^{2}$ Department of Mathematics, Bangladesh University of Engineering and Technology (BUET), BANGLADESH \\ ${ }^{*}$ Corresponding Contact: \\ Email: morshed5361@gmail.com
}

\begin{abstract}
In this paper, magnetohydrodynamic convection is analyzed numerically for a square enclosure filled with $\mathrm{Fe}_{3} \mathrm{O}_{4}$-water ferrofluid. A time-dependent exponential thermal boundary condition is applied at the bottom wall of the cavity. The ferrofluid is modeled as a single-phase fluid. Maxwell-Garnet model is used for modeling the effective thermal conductivity and viscosity of the ferrofluid. The Galerkin-weighted residuals method of finite-element analysis is adopted for the numerical solutions. The solid volume fraction, $\phi$ is varied from 2.5 to $10 \%$ and the Hartmann number Ha from 0 to 20 . Investigations are carried out for Rayleigh number $\mathrm{Ra}=10^{4}$ and $10^{5}$ over dimensionless times $\tau=0.01-1.0$. The present study indicates that $\mathrm{Ra}, \mathrm{Ha}$ and $\phi$, have a significant effect on heat transfer. At $\tau=1$, if $\mathrm{Ra}=10^{4}$, a higher solid volume fraction maximizes heat transfer whereas at $\mathrm{Ra}=10^{5}$, a lower solid volume fraction maximizes heat transfer. Moreover, at $\tau$ $=1$, incrementing $\mathrm{Ha}$ diminishes heat transfer at $\mathrm{Ra}=10^{4}$ whereas an optimum value of $\mathrm{Ha}=10$ maximizes heat transfer for $\mathrm{Ra}=10^{5}$. The exponential thermal boundary conditions have a certain importance on heat transfer. The present results provide necessary information for further investigation of heat transfer in its different applications.
\end{abstract}

Key words:

Ferrofluid, magnetohydrodynamic convection, exponential boundary condition, unsteady flow

\section{INTRODUCTION}

Heat transfer enhancement using nanofluids is gaining keen attention from researchers around the globe. Nanofluids have shown promising heat transfer characteristics compared with conventional fluids. Therefore, the application of nanofluid-based heat transfer has 
increased significantly in recent years. Nanofluids are used in for example solar thermal collectors, electronics coolers, and heat exchangers. Sometimes an external magnetic field is applied to control convection heat transfer properly. The magnetic field density therefore is important in different physical problems. This field influences the behavior of these nanofluids because of their magnetic susceptibility. All known nanofluids have so far shown no response when a magnetic field is applied to them. Nanofluids that are sensitive to magnetic fields are called magnetic nanofluids, their underlying convection mechanism changing significantly. Conventional fluids such as air and water are limited in their thermophysical properties (thermal conductivity, viscosity) in regard to heat transfer rate. Using metal, metal oxide, and carbonaceous material such as carbon nanotubes and graphene, a nanoparticle suspension in a convectional fluid was found to have promise as a working fluid that augmented the heat transfer rate significantly. These nanoparticle sizes varied from $0.1-100 \mathrm{~nm}$ and mixed in a certain solid volume fraction with the base fluid. Various nanofluids are used widely in practical applications such as solar thermal collectors, electronic coolers, and radiators. Still there is scope for new nanofluids to show better heat transfer rates. As a result, various investigations seek to make the thermal systems more efficient. Among the different nanofluids, $\mathrm{Cu}$-water, $\mathrm{Al}_{2} \mathrm{O}_{3}-$ water and $\mathrm{TiO}_{2}-$ water are common. They also have proven to be promising nanofluids in experimental studies. A few more emerging nanofluids have been reported that show a comparatively better heat transfer; included among these are $\mathrm{CNT}$-water, graphene-water, and $\mathrm{SiO}_{2}-$ water. These nanoparticles have been tried with different base fluids like water, ethylene glycol, methanol, and kerosene, all which show better heat transfer with a nanoparticle suspension.

Among the different cavity shapes, the square cavity has been widely investigated for heat transfer using nanofluid. Using homogenous and non-homogenous models, (Mansour and Sameh E. Ahmed, 2015) investigated the natural convection heat transfer in an inclined triangular enclosure filled with $\mathrm{Cu}$-water nanofluid saturated porous medium in the presence of heat generation effect. They concluded that good enhancement in the average Nusselt number can be obtained by an increase in the nanoparticle volume fraction. Augmentation of natural convection heat transfer in triangular shape solar collector by utilizing water based nanofluids having a corrugated bottom wall studied by (Rahman, et al., 2014). Numerical modeling for natural convection in partially C-shape open ended enclosure filled with nanofluid performed by (Bakier 2014). The author found that internal energy is related to cavity aspect ratio and the temperature all over the cavity increases as heat source length increases. (Das et al., 2015). studied the fully developed mixed convection flow in a vertical channel filled with nanofluids in the presence of a uniform transverse magnetic field. They found that the magnetic field tends to enhance the nanofluid velocity in the channel. (Sivasankaran and Pan, 2014) studied a square cavity filled with nanofluid for a sinusoidal temperature distribution and found the amplitude ratio, volume fraction of nanofluid, and Rayleigh number $R a$ enhance the heat transfer rate. (Abu Nada and Oztop, 2009) studied natural convection heat transfer in an inclined square cavity filled with a $\mathrm{Cu}$-water nanofluid and found that, at high $\mathrm{Ra}$, the Nusselt number $\mathrm{Nu}$ decreases proportionately. A similar problem is analyzed by (Mahmoudi et al., 2010) for Cu-water nanofluid and they found the solid volume fraction of the nanofluid enhances the heat transfer rate. Heat transfer enhancement by overlapped dual twisted-tapes (O-DTs) and $\mathrm{TiO} 2 /$ water nanofluids was experimentally and numerically investigated by (Eiamsa-ard et al., 2015). (Sandeep et al., 2016) analyzed the unsteady magnetohydrodynamic radiative flow and heat transfer characteristics of a dusty nanofluid over an exponentially permeable stretching surface in presence of volume fraction of dust and nano particles. (Rahman et al., 
2014) studied the effect of solid volume fraction and tilt angle in a quarter circular solar thermal collectors filled with CNT-water nanofluid. Fluid flow and heat transfer inside Lshaped enclosure filled with $\mathrm{Cu}$ /water nanofluid investigated numerically using the Lattice Boltzmann Method by (Bouchmel Mliki et al., 2015). (Krishnamurthy et al., 2016) studied the effect of chemical reaction on MHD boundary layer flow and melting heat transfer of Williamson nanofluid in porous medium. Effect of sine-squared thermal boundary condition on augmentation of heat transfer in a triangular solar collector filled with different nanofluids investigated by (Rahman et al., 2015). A study represents the heat transfer optimization of two-dimensional incompressible laminar flow of Al2O3-water nanofluids in a duct with uniform temperature corrugated walls conducted by (Valinataj-Bahnemiri et al., 2015). (Gireesha et al., 2016) investigated melting heat transfer in boundary layer stagnation-point flow of nanofluid toward a stretching sheet with induced magnetic field. (Aminossadati and Ghasemi, 2009) extended the problem for different nanofluids such as $\mathrm{Cu}$-water, $\mathrm{Al}_{2} \mathrm{O}_{3}$-water, $\mathrm{TiO}_{2}$-water, $\mathrm{Ag}$-water for the bottom heated wall of the square cavity and found nanofluid enhances heat transfer at low Ra. (Sameh E. Ahmed et al., 2016) investigated mixed convection from a discrete heat source in enclosures with two adjacent moving walls and filled with micropolar nanofluids. (Choi et al., 2014) investigated a square cavity filled with a $\mathrm{CuO}-$ water nanofluid. They concluded that incrementing the nanofluid solid volume fraction decreases the heat transfer rate because an increase in viscosity and a decrease in the coefficient of thermal expansion and specific heat. (Sivasankaran and Pan, 2014) studied a square cavity filled with nanofluid for a sinusoidal temperature distribution and found the amplitude ratio, volume fraction of nanofluid, and Rayleigh number $R a$ enhance the heat transfer rate. Abu Nada and Oztop (2009) studied natural convection heat transfer in an inclined square cavity filled with a $\mathrm{Cu}$-water nanofluid and found that, at high $R a$, the Nusselt number $N u$ decreases proportionately. A similar problem is analyzed by (Mahmoudi et al., 2010) for $\mathrm{Cu}$-water nanofluid and they found the solid volume fraction of the nanofluid enhances the heat transfer rate. Similar results are found by (Jahansahi et al., 2010) for a $\mathrm{SiO}_{2}$-water nanofluid. (Aminossadati and Ghasemi, 2009) extended the problem for different nanofluids such as $\mathrm{Cu}$-water, $\quad \mathrm{Al}_{2} \mathrm{O}_{3}$-water, $\mathrm{TiO}_{2}$-water, $\mathrm{Ag}$-water for the bottom heated wall of the square cavity and found nanofluid enhances heat transfer at low $\mathrm{Ra}$. (Ho et al., 2008) also studied square cavities filled separated with $\mathrm{CuO}-$ water and $\mathrm{Al}_{2} \mathrm{O}_{3}$-water nanofluid for which the left-side vertical wall was heated and concluded that effective viscosity and thermal conductivity of nanofluids are key in obtaining better heat transfer. For a square cavity filled with $\mathrm{Cu}$-water nanofluid, (Santra et al., 2008) suggested selected values of $R a$ and solid volume fraction ensure a better heat transfer.

Ferrofluids, also referred to as magnetic nanofluids, have a relatively strong magnetic susceptibility and a greater potential in augmenting heat transfer in magnetohydrodynamic (MHD) convection. While not a recent discovery, they are age-old nanofluids whose potential has been largely overlooked; being also very economical, their field of application has been extended. Different potential ferrofluids have been reported recently. Among them cobalt, nickel, magnetite $\left(\mathrm{Fe}_{3} \mathrm{O}_{4}\right)$, ferric oxide and ferro-zinc magnetite are used as ferromagnetic particles. These magnetic particles are used with different base fluids such as water, kerosene, and ethylene glycol. (Seikholeslemi and Gorji, 2014) recently analyzed free convection of a nickel ferrofluid in a square cavity, which was heated from below and subjected to a magnetic field. They concluded that a larger solid volume fraction for the nanoparticles in the base fluid provides better heat transfer. (Rabbi et al., 2010) recently investigated mixed convection in ferrofluid filled lid driven cavity in different heater configuration and found that at higher $R i$ and lower $\mathrm{Ha}$, semi-circular notched cavity shows significantly better (more than $30 \%$ ) heat 
transfer rate. (Kefayati, 2014) studied natural convection in a linearly heated cavity using the Lattice Boltzmann method (LBM) and showed that an external magnetic field has a significant effect on heat transfer and higher $R a$ ensures higher heat transfer. (Ashouri et al., 2010) studied pure magnetic convection and established a correlation numerically for $\mathrm{Nu}$ in a side-wall-heated square cavity filled with ferrofluid. (Jue, 2006) analyzed combined thermal and magnetic convection in a ferrofluid filled square cavity using a semi-implicit finite-element method. For the cavity, the side walls are kept at different temperatures and the position of magnetic field was at the bottom. The author concluded that a suitable location and orientation of the magnetic field is useful for enhancing heat transfer in practical applications. (Ghasemian et al., 2015) investigated laminar heat transfer in a mini-channel for a $\mathrm{Fe}_{3} \mathrm{O}_{4}$ ferrofluid under constant and alternating magnetic fields. They reported that for an alternating magnetic field, heat transfer is enhanced to $27.72 \%$ whereas a constant magnetic field enhances heat transfer to $16.48 \%$ at a particular Reynolds number. (Sheikholeslami et al., 2015) studied a semi-annulus filled with a ferrofluid in the presence of a magnetic field. They used a control volume-based finite-element method and concluded that $N u$ is an increasing function of $R a$, solid volume fraction of ferrofluid, and magnetic number whereas heat transfer decreases with Hartmann number $\mathrm{Ha}$ and radiation parameter. (Bozhko et al., 2007) investigated wave ferrofluid convection both experimentally and numerically and found that the ferrofluid has a significant effect on flow phenomena when a magnetic fieldis present. (Aminfar et al., 2013) studied numerically the ferrofluid ( $\mathrm{Fe}_{3} \mathrm{O}_{4}$-water) heat transfer in a rectangular duct and concluded that with increasing Reynolds number, the effect of traverse magnetic field is more profound than axial non-uniform magnetic field. (Belyaev and Smorodin, 2010) studied the stability of ferrofluid when a magnetic field is present. Applying the finite-element model, (Snyder et al., 2003) studied magnetoconvection in ferrofluid in a cubic enclosure. (Selimefendigil et al., 2014) studied natural convection in a partially heated square enclosure filled with a ferrofluid and found that the midvertical position of the heater yields a better heat transfer. (Selimefendigil and Oztop, 2014) studied forced convection in a vented cavity having a rotating cylinder for ferrofluid and found that the rotational speed of the cylinder enhances heat transfer.

MHD convection in magnetic nanofluids behaves differently compared with conventional fluids. Basically, the nanoparticles and base fluid are electrically conductive. When this magnetic nanofluid moves in the present of a strong external magnetic field, it creates a Lorentz force. The coupling between this Lorentz force and the buoyancy force gives rise to convection. A strong magnetic field suppresses the fluid motion and hence decreases the heat transfer rate. This application of the magnetic field can be key in controlling the convection where necessary. Also, the orientation and inclination angle of the applied magnetic field have a certain effect on heat transfer. Studying the effect of heat dissipation on MHD convection in an inclined cavity filled with cobalt-kerosene ferrofluid, (Kefayati, 2014) found that the magnetic field affects the heat transfer for a particular Rayleigh number. (Mahmoudi et al., 2013) studied MHD natural convection and entropy generation for a trapezoidal cavity filled with $\mathrm{Cu}$-water nanofluid. They found at low $R a\left(R a=10^{4}-10^{5}\right)$, incrementing $\mathrm{Ha}$ enhances the heat transfer but, at higher $\mathrm{Ra}$, heat transfer decreases with Ha. Using LBM, (Sheikhholeslami, 2014) studied MHD natural convection and found $N u$ increases with $R a$ and solid volume fraction but decreases with $\mathrm{Ha}$. A similar result is reported by (Zhou and Yan, 2015). They also reported a critical $R a$ and orientation of the magnetic field for maximum heat transfer rate.

In practice, different kinds of heat generation are observed depending on the thermal boundary conditions for the cavity. In general, heating can be classified as isothermal or non-isothermal. (Mejri et al., 2015) studied MHD natural convection in a nanofluid-filled open cavity with a sinusoidal boundary condition and they reported incrementing $\mathrm{Ha}$ 
decreases the heat transfer for the nanofluid. (Wu et al.,2015) studied natural convection for porous rectangular cavity having sinusoidal temperature distribution in both side walls and they found a sinusoidal thermal boundary condition enhances the heat transfer significantly. Recently, (Rahman et al., 2015) studied unsteady heat transfer in a square cavity filled with CNT-water nanofluid having non-isothermal bottom heater. They concluded the nanofluid allows for an optimum enhancement of heat transfer.

The current literature indicates that $\mathrm{Ra}, \mathrm{Ha}$, the inclination angle of the cavity, cavity shape, solid volume fraction of ferrofluid, and dimensionless time are the key parameters for the present investigation. Increasing $R a$ can enhance heat transfer due to the active buoyancy force, which diminishes with the application of the Lorentz force created by the external magnetic field. In contrast, a ferrofluid adds contributions depending on its thermal conductivity and dynamic viscosity, which can be used to augment heat transfer rate.

Among the different non-isothermal heating, sinusoidal and position-dependent thermal boundary conditions have been previously analyzed. However, a time-dependent exponential-type thermal boundary condition has not been analyzed despite being very common in real-life application. Hence, we analyzed numerically the effect of a time-dependent thermal boundary condition on heat transfer in the presence of external magnetic field. For its higher magnetic susceptibility, a ferrofluid is chosen to act as working fluid. In this paper, unsteady heat transfer is investigated to understand its underlying mechanism properly. The solid volume fraction of the ferrofluid, $R a$ and $\mathrm{Ha}$ are considered pertinent parameters and their effect on heat transfer is analyzed by following their dimensionless-time evolution. The present investigation can be applied to design more realistic heat transfer equipment as we consider exponential-type time-dependent heating.

\section{Formulation of the Problem}

\section{Theoretical model}

A square enclosure having length and width $L$ is considered (Fig. 1). The cavity is filled with $\mathrm{Fe}_{3} \mathrm{O}_{4}$-water ferrofluid. The bottom wall of the enclosure is heated and its temperature at the boundary varies exponentially with time. The top wall of the cavity is kept at a low temperature $\left(T=T_{c}\right)$ whereas on the left and right-vertical walls the thermal conditions are adiabatic. The entire boundary is assumed to be stationary. A Cartesian co-ordinate system is defined (Fig. 1).

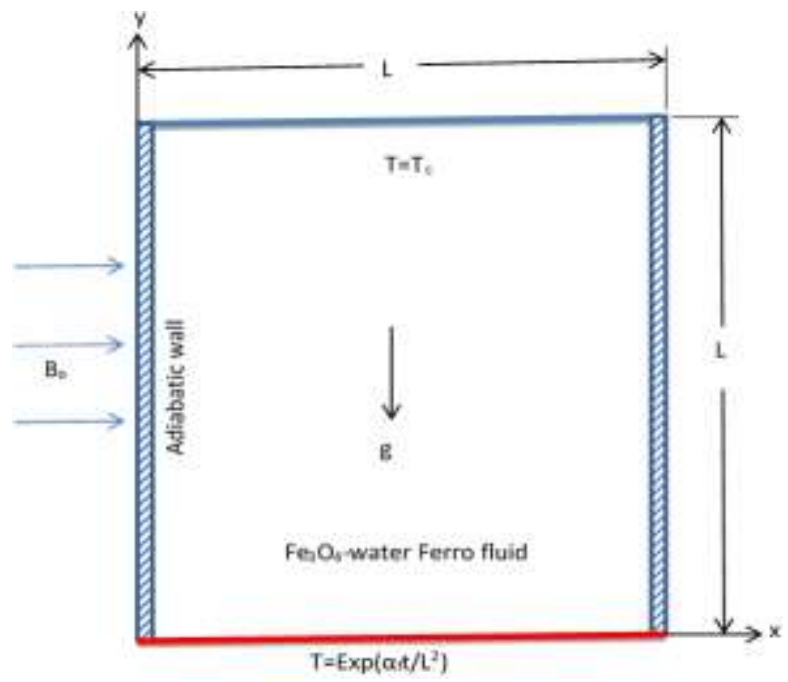

Fig. 1: Schematic view of the cavity with the boundary conditions 
Acceleration due to gravity is directed along the negative $y$ axis. A magnetic field with magnetic flux density $B_{o}$ is applied horizontally on the left of the enclosure. Given boundary conditions describing time-dependent exponential heating, the bottom wall gradually heats as time elapses. To capture the unsteady flow and thermal field behavior, a dimensionless time $\tau$ is introduced and varies widely in value. Radiation heat transfer and viscous heating are neglected for the present analysis.

Time-dependent exponentially varying boundaries are realistic from the point of view of applications. In practice, heating goes up exponentially in many heat-transfer problems. For example, the current flowing through an electrical diode varies exponentially; the heat generated is proportional to the square of the current and proportional to time, hence the exponential heat generation. In electronic heat transfer, there is also the presence of a strong magnetic field. To study the time-dependent behavior of such heat transfers, the following analysis is useful.

\section{Mathematical Modeling}

The two-dimensional unsteadycontinuity,momentum, and energy equations are used to model flow and thermal fields of the problem. The working fluid is assumed to be an incompressible Newtonian fluid with constant thermophysical properties, except density, and a thermal equlibrium is assumed between the ferromagnetic particles and the base fluid. The Boussinesq approximation is employedfor the density variation of the fluid. The dimensionless governing equations then take the form

$$
\frac{\partial(\delta)}{\partial \tau}+\frac{\partial(U \delta)}{\partial X}+\frac{\partial(V \delta)}{\partial Y}=\frac{\partial}{\partial X}\left(\Gamma_{\delta} \frac{\partial \delta}{\partial X}\right)+\frac{\partial}{\partial Y}\left(\Gamma_{\delta} \frac{\partial \delta}{\partial Y}\right)+S_{\delta}
$$

Here, dimensionless dependent variables are designated by $\delta$ and the corresponding diffusion and source terms are denoted by $\Gamma_{\delta}$ and $S_{\delta}$, respectively (Table 1).

Table 1: Summary of terms in the non-dimensional governing equations (1)

\begin{tabular}{cccc}
\hline Equations & $\delta$ & $\Gamma_{\delta}$ & $S_{\delta}$ \\
\hline Continuity & 1 & 0 & 0 \\
$U$-momentum & $U$ & $\mu_{n f} / \rho_{n f} \alpha_{f}$ & $-\partial P / \partial X$ \\
$V$-momentum & $V$ & $\mu_{n f} / \rho_{n f} \alpha_{f}$ & $-\partial P / \partial Y+(\rho \beta)_{n f} /\left(\beta_{f} \rho_{n f}\right) \operatorname{Ra} \operatorname{Pr} \Theta-\left(\sigma_{n f} \rho_{f}\right) /\left(\rho_{n f} \sigma_{f}\right) H a^{2} \operatorname{Pr} V$ \\
Thermal energy & $\Theta$ & $\alpha_{n f} / \alpha_{f}$ & 0 \\
\hline
\end{tabular}

The definitions of the various scales that are adopted to obtain the above equations are

$$
X=\frac{x}{L}, Y=\frac{y}{L}, \tau=\frac{\alpha_{f} t}{L^{2}}, U=\frac{u L}{\alpha_{f}}, V=\frac{v L}{\alpha_{f}}, P=\frac{\left(p+\rho_{f} g y\right) L^{2}}{\rho_{f f} \alpha_{f}^{2}}, \Theta=\frac{\left(T-T_{c}\right)}{\left(T_{h}-T_{c}\right)} .
$$

Where $U, V, P$, and $\Theta$ are the dimensionless velocity, pressure, and temperature, respectively. Here, subscripts ' $f f$ ' and ' $f$ ' refer to ferrofluid and base fluid, respectively.

The dimensionless parameters $R a$, Prandtl number Pr, and Hacan be defined as

$$
R a=\frac{g \beta_{f} L^{3}\left(T_{h}-T_{c}\right)}{\alpha_{f} v_{f}}, \operatorname{Pr}=\frac{v_{f}}{\alpha_{f}}, H a=B_{o} L \sqrt{\frac{\sigma_{f}}{\mu_{f}}},
$$

Where $\sigma_{f}$ denotes the electrical conductivity of the fluid. The density of ferrofluid, which is assumed to be constant, can be expressed as 


$$
\rho_{f f}=(1-\phi) \rho_{f}+\phi \rho_{s} \text {. }
$$

Here the quantities referring to the solid ferromagnetic particles (magnetite) are indicated by subscript ' $s$ '. In the above equation, the solid volume fraction $\phi$ has a significant effect on the thermal diffusivity of the ferrofluid, which is quite different to that of the base fluid, and can be modeled as

$$
\alpha_{f f}=\frac{k_{f f}}{\left(\rho c_{p}\right)_{f f}}
$$

where the heat capacitance of the ferrofluid $\left(\rho c_{p}\right)_{f f}$ is given by

$$
\left(\rho c_{p}\right)_{f f}=(1-\phi)\left(\rho c_{p}\right)_{f}+\phi\left(\rho c_{p}\right)_{s} .
$$

In addition, the thermal expansion coefficient $\beta_{f f}$ of the ferrofluid follows from

$$
(\rho \beta)_{f f}=(1-\phi)(\rho \beta)_{f}+\phi(\rho \beta)_{s} \text {. }
$$

Moreover, the dynamic viscosity $\mu_{f f}$ of the ferrofluid can be expressed using the (Brinkman model, 1952)

$$
\mu_{f f}=\frac{\mu_{f}}{(1-\phi)^{2.5}} \text {. }
$$

The effective thermal conductivity of the ferrofluid can be described using the (MaxwellGarnet model, 1904)

$$
\frac{k_{f f}}{k_{f}}=\frac{k_{s}+2 k_{f}-2 \phi\left(k_{f}-k_{s}\right)}{k_{s}+2 k_{f}+\phi\left(k_{f}-k_{s}\right)}
$$

and the effective electrical conductivity of the ferrofluid can be described using the (Maxwell model,1904)

$$
\frac{\sigma_{f f}}{\sigma_{f}}=1+\frac{3\left(\sigma_{s} / \sigma_{f}-1\right) \phi}{\left(\sigma_{s} / \sigma_{f}+2\right)-\left(\sigma_{s} / \sigma_{f}-1\right) \phi} .
$$

The initial and boundary conditions defined at $\tau=0$ are in dimensionless form,

Entire domain: $U=V=0, \Theta=0$, and $\tau>1$,

On the vertical wall: $U=V=0, \frac{\partial \Theta}{\partial X}=0$

On the top horizontal wall: $U=V=0, \Theta=0$.

On the bottom horizontal wall: $U=V=0, \Theta=e^{\tau}$

The local $N u$ on the heat source surface can be expressed as $N u_{L}=-\frac{k_{f f}}{k_{f}} \frac{\partial \Theta}{\partial Y}$. The average $N u$ is evaluated by integrating $N u$ L along the heat source,

$$
N u_{a v}=-\frac{k_{f f}}{k_{f}} \int_{0}^{1} \frac{\partial \Theta}{\partial Y} d X .
$$


The flow field of the present problem is visualized through streamlines which are obtained from the stream function. The stream function is defined from the velocity components $U$ and $V$, the relationship for two-dimensional flows being

$U=\frac{\partial \Psi}{\partial Y}, V=-\frac{\partial \Psi}{\partial X}$

\section{Ferrofluids}

Ferrofluids are mainly nanofluids for which ferromagnetic particles are used. As they exhibit a magnetic susceptibility, magnetic fields interact strongly with them. Different ferrofluids are being tested for their heat transfer properties. Among them, Co-kerosene, $\mathrm{Ni}$-water, $\mathrm{Fe}_{3} \mathrm{O}_{4}$-water, $\mathrm{Fe}_{2} \mathrm{O}_{3}$-water are quite common known. The present investigation uses $\mathrm{Fe}_{3} \mathrm{O}_{4}$-water ferrofluid, which is modeled as a single-phase fluid using the MaxwellGarnet model. The thermophysical properties of theFe $\mathrm{O}_{4}$ ferromagnetic particle and water (base fluid) is presented in Table 2.

Table 2: Thermophysical properties of water and $\mathrm{Fe}_{3} \mathrm{O}_{4}$

\begin{tabular}{cccccc}
\hline & $\begin{array}{c}c_{p} \\
\left(\mathrm{Jkg}^{-1} \mathrm{~K}^{-1}\right)\end{array}$ & $\begin{array}{c}\rho \\
\left(\mathrm{kgm}^{-3}\right)\end{array}$ & $\begin{array}{c}k \\
\left(\mathrm{Wm}^{-1} \mathrm{~K}^{-1}\right)\end{array}$ & $\begin{array}{c}B \\
\left(\mathrm{~K}^{-1}\right)\end{array}$ & $\begin{array}{c}\boldsymbol{\Sigma} \\
\left(\text { Siemens } \mathrm{m}^{-1}\right)\end{array}$ \\
\hline Water & 4179 & 997.1 & 0.613 & $2.1 \times 10^{-4}$ & 0.05 \\
$\mathrm{Fe}_{3} \mathrm{O}_{4}$ & 670 & 5200 & 6 & $1.3 \times 10^{-5}$ & 25,000 \\
\hline
\end{tabular}

\section{Numerical Methodology}

\section{Numerical Schemes}

The Galerkin-weighted residuals method of finite-element analysis is adopted to obtain a numerical solution of the present problem. The entire domain is first discretized into several elements. These elements form the mesh for the geometry and the boundary wall. Here a six-nodded triangular mesh element is chosen. The elements are of arbitrary shape. The key idea of the solution algorithm proposed in present work is to consider the two momentum equations for two-dimensional laminar flow and solve both velocity components. The equation of continuity is used in solving the pressure, whereas the energy equation is used for solving the temperature. A fine boundary mesh is applied to avoid singularities that can arise at corner points. The specified boundary conditions are applied to the governing equation to formulate the nonlinear algebraic equations set. Later a solution matrix is formulated for all dependent variables. An iterative technique is adopted to solve the matrix. A limit threshold of $10^{-6}$ is set as a convergence criterion for all dependent variables.

\section{Grid Test}

The number of elements in the mesh affects the numerical solution. Hence a grid test is performed to ensure the numerical accuracy for the present problem. Fig. 2 shows the grid test result for $R a=10^{5}, H a=10$, and a solid volume fraction of $10 \%$. The average $N u$ is plotted against dimensionless time $\tau$. From the figure, it is clear that average $N u$ varies significantly for 4844elements. After we reached a grid having 10258 elements, there is no significant variation in the average $N u$. Therefore, setting the number of elements to 4844 as our standard, this size of grid reduces the computational time and cost without hampering the accuracy of numerical results. 


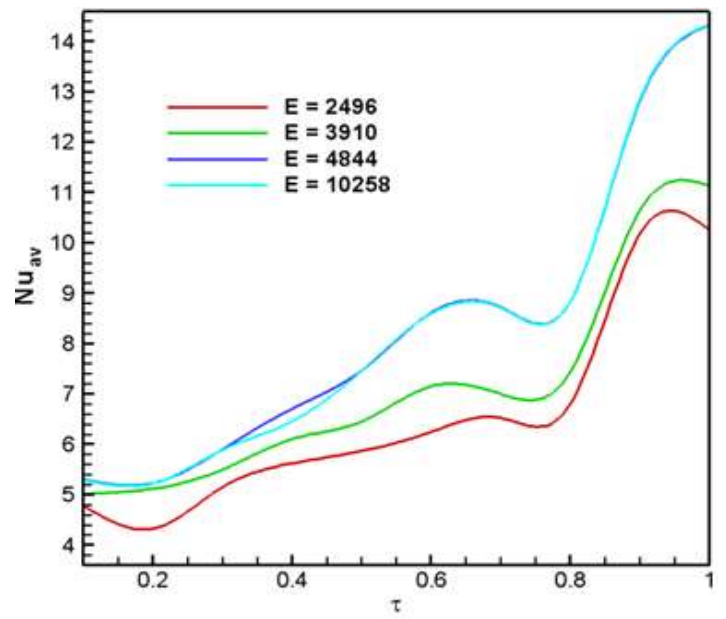

Fig. 2: Grid independency study with $\varphi=0.04, \mathrm{Ha}=10$ and $\mathrm{Ra}=10^{5}$

\section{Code Verification}

The code of this present investigation is verified with the previous numerical study of (Mejri et al., 2014) for square cavity filled with $\mathrm{Al}_{2} \mathrm{O}_{3}$-water nanofluid. The results from this verification are presented through illustrations of streamlines, isotherm contours. Comparison of the flow field and the thermal field is shown in Fig. 3 for $\mathrm{Ra}=5 \times 10^{4}, \mathrm{Ha}=50$, and $\varphi=0$ and 0.04 . From the figure, the present code is in good agreement with the established literature of (Mejri et al., 2014). Therefore, the present numerical method is reliable.
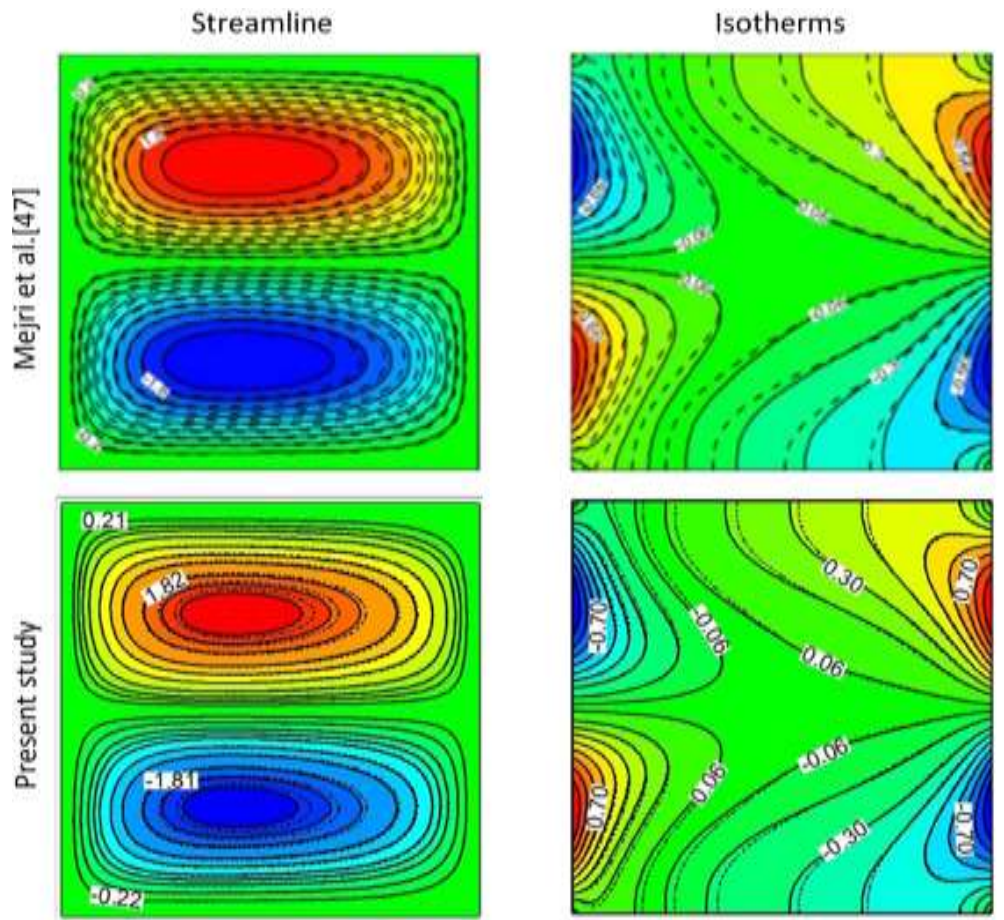

Fig 3: Comparison of streamline and isotherm contours for $\mathrm{Ra}=5 \times 10^{4}, \mathrm{Ha}=50$ between numerical results by Mejri et al. (2014) and present study 


\section{RESULTS AND DISCUSSION}

In the present study, the effect of solid volume fraction and magnetic field is presented for unsteady convection. $\mathrm{Fe}_{3} \mathrm{O}_{4}-$ water is considered as a single-phase ferrofluid; its solid volume fraction is varied from $2.5-10 \%$. The value of $\mathrm{R} a$ was set to $10^{4}$ and $10^{5}$, and $\mathrm{Ha}$ was allowed to vary from 0-20. All the simulations are performed for dimensionless time $\tau=0.01-1.00$. In this section, the results are discussed based on streamlines, isotherm contours plot along with stream functions, and the local and average $N u$.

\section{Effect of Solid Volume Fraction of Ferrofluid}

In Fig. 4, the effect of solid volume fraction on streamlines are presented for different solid volume fractions at $R a=10^{4}, H a=10$, and $\tau=0.1$ and 1.0. For $\tau=0.1$, the streamline patterns are irregular and the value of $\psi_{\max }=0$. This is quite obviously due to the exponential timedependent boundary condition.
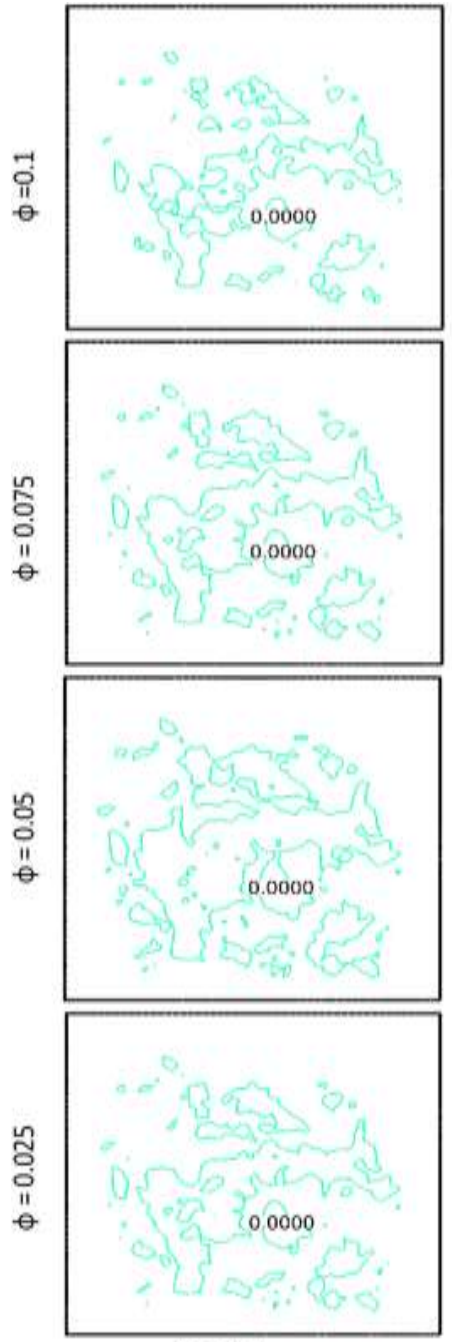

$\tau=0.1$
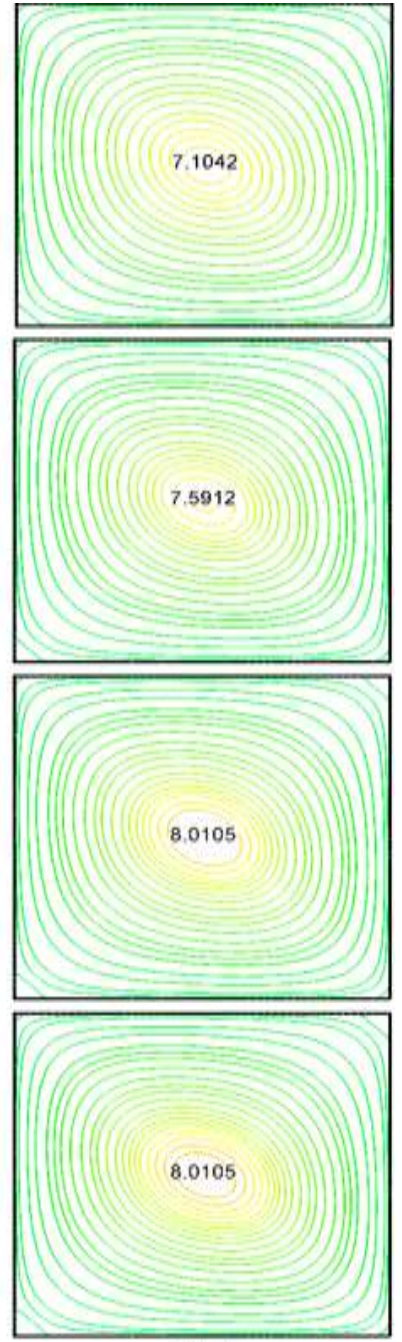

$\tau=1$

Fig. 4: Effect of solid volume fraction on streamlines for the selected values of $\tau$ with $\mathrm{Ha}=$ 10 and $\mathrm{Ra}=10^{4}$ 
The unsteady nature of the convection reveals that no streamline is generated in such short times. Incrementing the solid volume fraction does not show any significant effect for times $\tau=0.1$, although the irregular streamline pattern is changed. Nevertheless, it still does not mean there is a convection pattern. For $\tau=1$, a clockwise circulation of streamline pattern is found inside the cavity. At this value of $\tau$, near the bottom wall of the cavity, convection is isothermal and at a higher temperature than that near the top wall. As a result, strong convection occurs because of this temperature difference. The fluid is heated at the heated bottom wall, then pushed upwards by the buoyancy force, and finally returned after transferring heat to the cold top wall. This creates a strong eddy, positioned in the middle of the cavity. As the value of $\phi$ increases from 0.025 to 0.1 , the value of stream function diminishes.
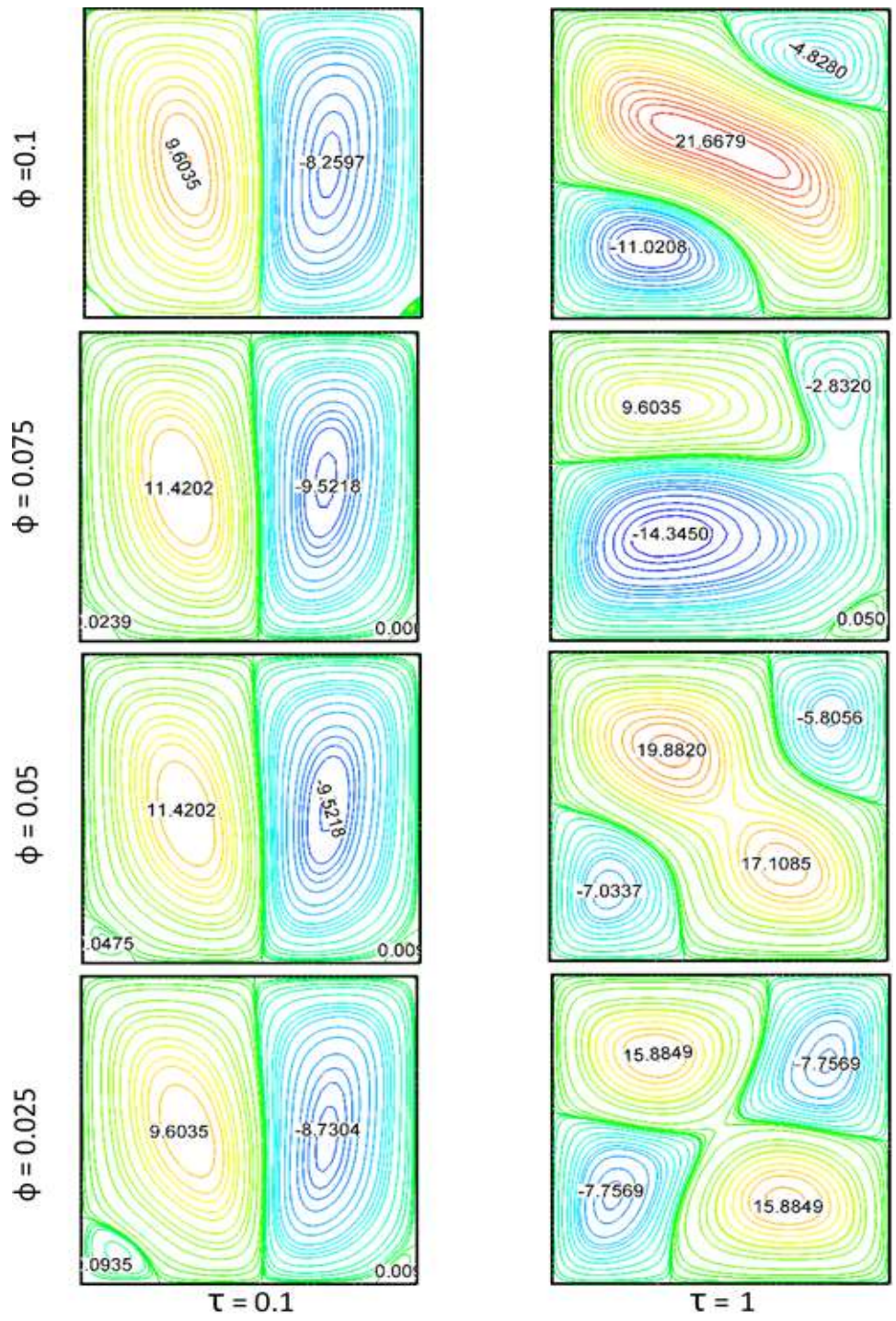

Fig. 5: Effect of solid volume fraction on streamlines for the selected values of $\tau$ with $\mathrm{Ha}=$ 10 and $\mathrm{Ra}=10^{5}$ 
Fig. 5 shows the streamline for $R a=10^{5}$ for different solid volume fractions at $\tau=0.1$ and 1.0. For $\tau=0.1$, two major symmetric cells can be found inside the cavity. There is a minor vortex in the lower-left region of the cavity. Two major symmetric vortices oppose each other in the pattern. The value of the stream function for these vortices increases to $\phi=0.05$ and then decreases again. For $\phi=0.1$, the lower-left vortex has vanished. For $R a=10^{5}$ and $\tau=1$, the minor vortices are elongated and cover a major region of the cavity. For $\phi=0.025$, two anticlockwise-rotating vortices counter two clockwise-rotating vortices at upper-left and lower-right of the cavity. With a further increment in $\phi(\phi=0.05)$, the strength of the anticlockwise-rotating vortices diminishes whereas the strength of the clockwise-rotating vortices enhanced.
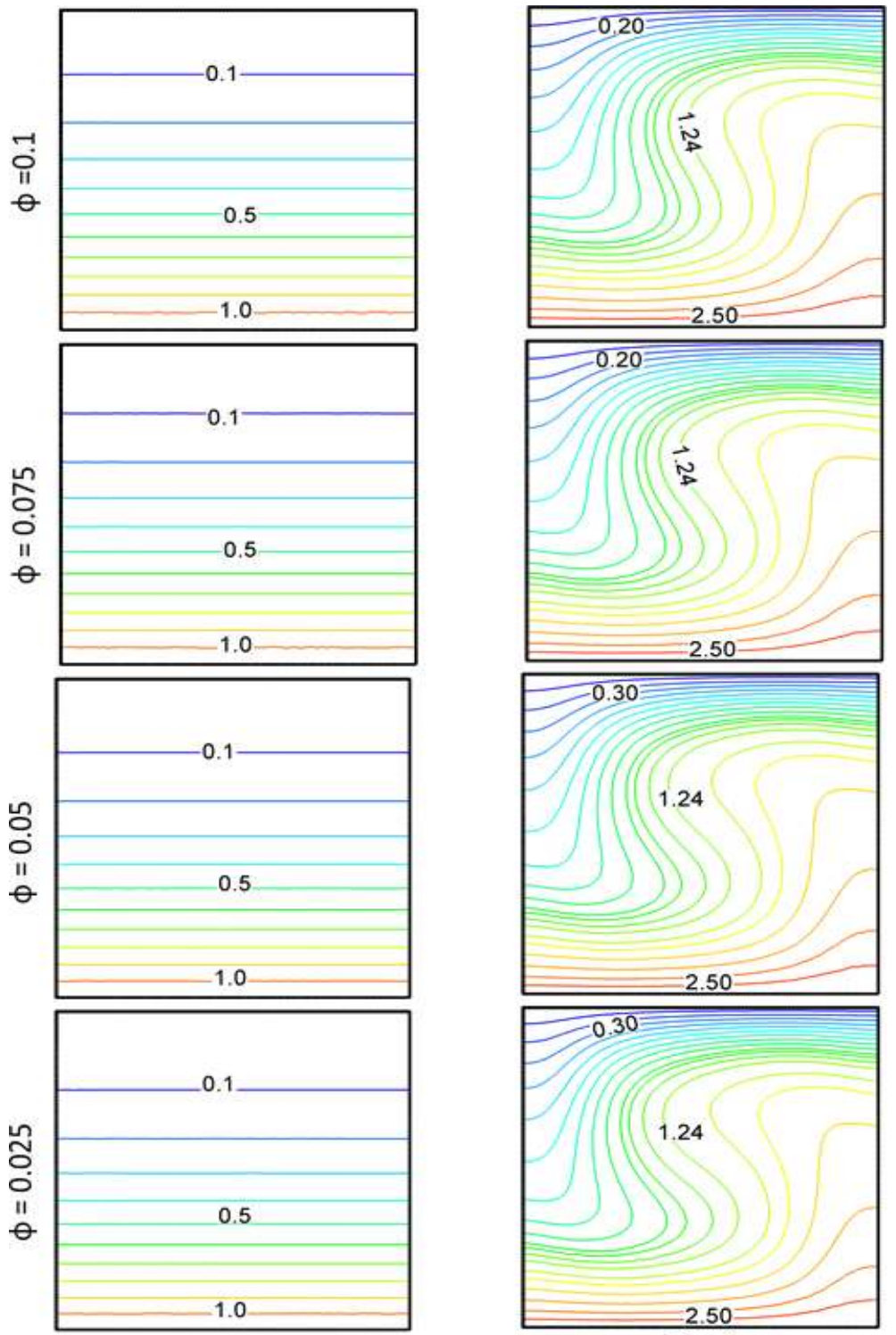

$$
\tau=0.1
$$

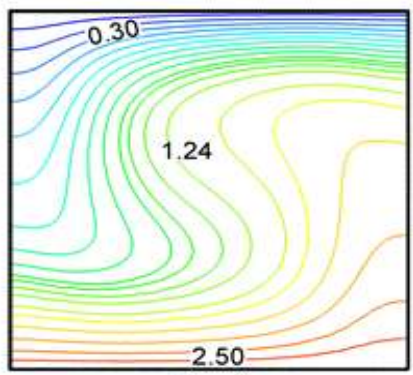

$\tau=1$

Fig. 6: Effect of solid volume fraction on isotherms for the selected values of $\tau$ with $\mathrm{Ha}=10$ and $\mathrm{Ra}=10^{4}$ 
The vortices no longer remain symmetric. For $\phi=0.075$, there remain two major vortices rotating in countering directions. Situated near the left-bottom and right-bottom corners, the other two vortices are weaker in nature. For $\phi=0.1$, one vortex weakens and there remains only three vortices inside the cavity, the clockwise rotating vortex being the strongest.

From Figs. 4 and 5, one notes a significant effect of $R a$ on streamlines. With increasing $R a$, the temperature difference between the bottom and top walls of the cavity increased. With $R a=10^{5}$, due to the time-dependent exponential boundary condition, it takes a longer time to arrive at the steady state. The fluid inside the cavity then tries to increase the temperature of the cold wall. The solid volume fraction of the ferrofluid determines largely the flow pattern of the fluid at $\tau=1$. For $\tau=1$, the bottom wall is heated more than for $\tau=0.1$, and hence also causes a significant variation in the flow behavior between these two cases.
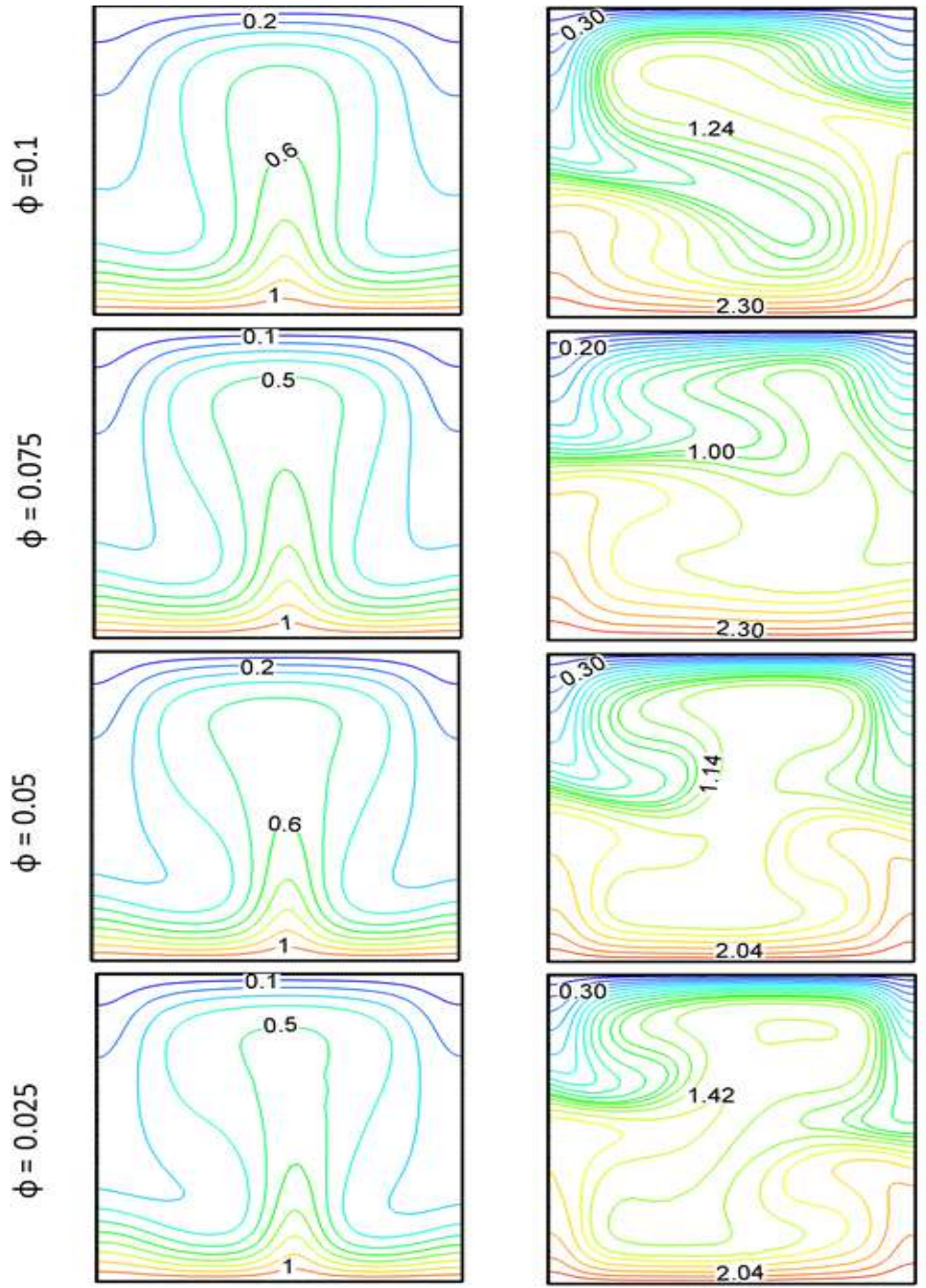

$$
\tau=0.1
$$

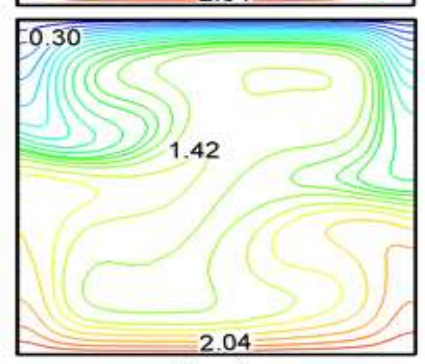

$$
\tau=1
$$

Fig. 7: Effect of solid volume fraction on isotherms for the selected values of $\tau$ with $\mathrm{Ha}=10$ and $\mathrm{Ra}=10^{5}$ 
Fig. 6 shows for $\tau=0.1$ and 1 the isotherms for different solid volume fractions of the ferrofluid. Note that the isotherms are parallel to each other at $\tau=0.1$. Also, the temperature of the bottom wall is lower than that for $\tau=1$. At $\tau=0.1$ and $R a=10^{4}$, no streamline pattern gets established. Therefore, the fluid is static-like and no fluid convection takes place. All heat transfer is by conduction within the fluid layer. A higher solid volume fraction of ferrofluid does not improve the degree of convection. If $\tau=1$, there is a clockwise-rotating fluid motion, which creates a thermal gradient near the bottom wall and the isotherms are found from the thermal boundary layer. Incrementing the solid volume fraction from 0.0251.0 shows no significant variation for these isotherms at $\tau=1$. For $R a=10^{5}$ and $\tau=0.1$, heat transfer is mainly by convection (Fig. 7).

(c)

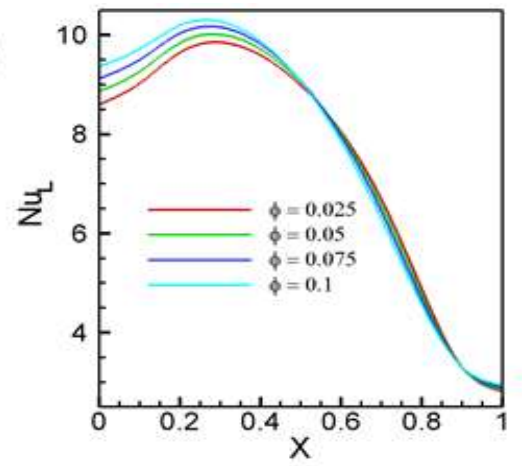

(b)

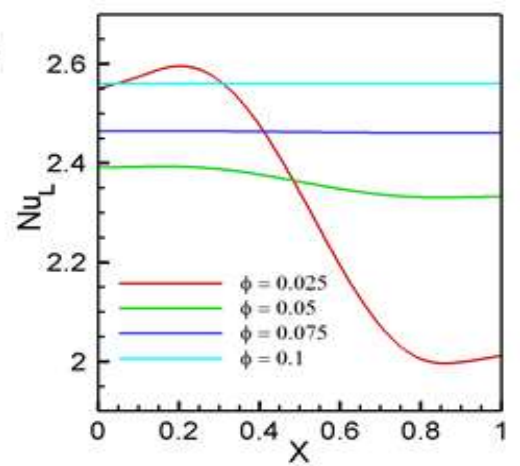

(a)

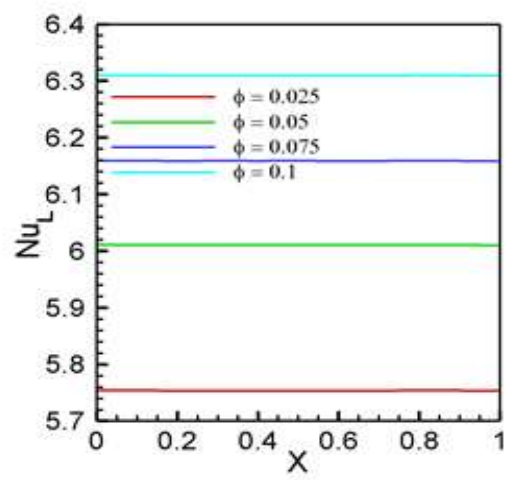

$\mathrm{Ra}=10^{4}$
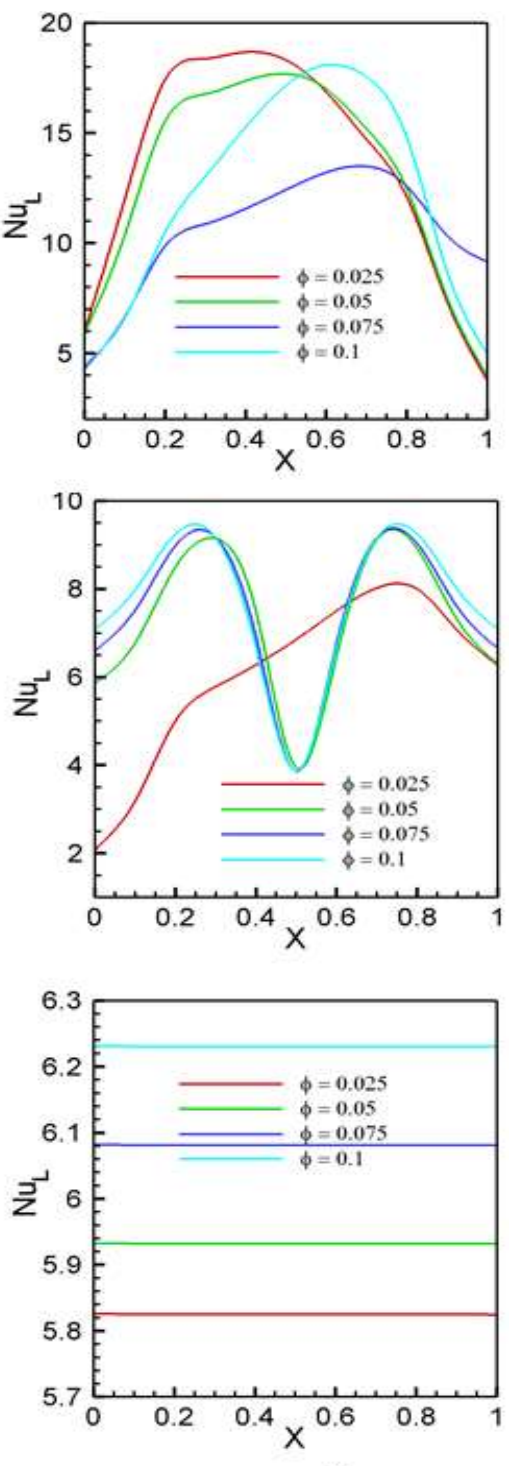

$\mathrm{Ra}=10^{5}$

Fig. 8: Effect of the nanoparticle volume fraction and dimensionless time on local Nusselt number at $\mathrm{Ha}=10$ and (a) $\tau=0.01$, (b) $\tau=0.5$ and (c) $\tau=1$ 
The isotherm pattern is plume like and the patterns for different solid volume fractions are almost similar. A noticeable change occurs at $\tau=1$, for which the isotherms indicate strong convection. A thermal boundary layer forms near the cavity wall with a higher thermal gradient than for $\tau=0.1$. The isotherms are spread over a greater extent in comparison with those for $R a=10^{4}$.

Fig. 8 gives the local $N u$ distribution for $R a=10^{4}$ and $10^{5}$ at different dimensionless times and solid volume fractions. For $\tau=0.01$, convection occurs at a very early stage. For both $R a$ $=10^{4}$ and $10^{5}$, the local $N u$ distribution is constant at the heated bottom boundary. For this value of $\tau$, the local $N u$ is higher for higher solid volume fraction. This phenomenon is quite obvious as no fluid motion is established at this very early stage of convection. As a result, heat transfer is by conduction creating a uniform $N u$ distribution over the heated wall. For $\tau=0.5$, this uniformity changes. A higher solid volume fraction yields higher local $N u$. At low solid volume fraction $(\phi=0.025)$, the local $N u$ reaches a maximum and later decreases over the right-side wall. However, for higher solid volume fraction, the local $N u$ remains uniform and produces a higher ferrofluid momentum. As $R a$ is low $\left(R a=10^{4}\right)$, the buoyancy force produced under the temperature difference between the cold and hot boundary walls is low. Therefore, ferrofluid having low momentum (for $\phi=0.025$ ) can move easily. At higher solid volume fraction, the fluid motion is not that easy and causes conduction but still ensures higher $N u$ because of higher thermal conductivity. For $R a=10^{5}$, the local $N u$ is relatively higher than $R a=10^{4}$. For this case (at $\tau=0.5$ ) due to the higher $R a$, the buoyancy force acting is higher. Therefore, a strong convection occurs for higher solid volume fraction. The local $N u$ is higher near $x=0.25$ and 0.75 . The curves form a symmetric pattern. However, little variation is observed for $\phi=0.05,0.75$, and 0.1 . For $\phi=0.025$, the local $N u$ is much lower. For $\tau=1$, we find that the local $N u$ is maximum near $x=0.3$. After that a sharp fall is noticeable. Here, higher solid volume fraction ensures better heat transfer. For $R a=10^{5}$, an interesting variation in the local $N u$ can be observed; a maximum can be found for lower solid volume fraction $(\phi=0.025)$. For $\phi=0.075$, the local $N u$ is much lower compared with other values of solid volume fraction.

(b)

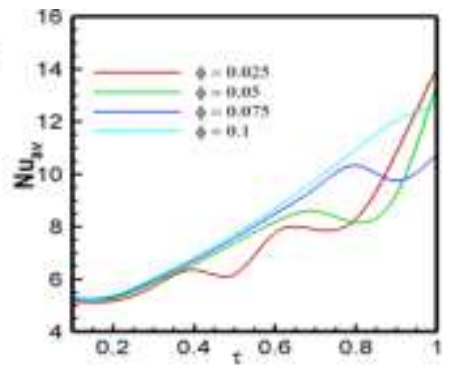

(a)

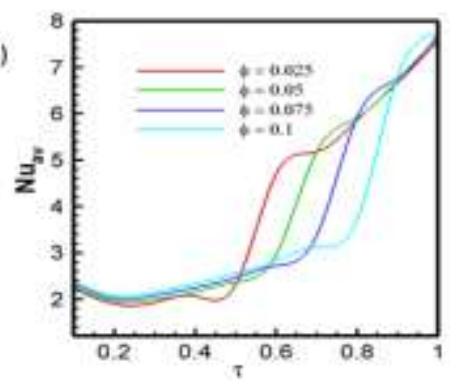

Fig. 9: Effect of the nanoparticle volume fraction on average Nusselt number at $\mathrm{Ha}=10$ and (a) $\mathrm{Ra}=10^{4}$, (b) $\mathrm{Ra}=10^{5}$ 
Fig. 9 shows the average $N u$ at the heated bottom wall for different dimensionless times. For $R a=10^{4}$, there is a jump in $N u$ at different value of $\tau$ for different solid volume fractions. Initially the value of average $N u$ decreases with dimensionless time. After $\tau=0.2$, the value of the average $N u$ increased. With further increments in $\tau$, the first jump in $N u$ occurs for $\phi$ $=0.025$. It is found that higher solid volume fraction needs longer times for the jump. At $\tau$ $=1$, higher solid volume fraction shows maximum average $N u$. A similar jump is seen for $R a=10^{5}$, but this jump occurs for a lower solid volume fraction only. For $R a=10^{5}$ and at $\tau=$ 1 , lower solid volume fractions can produce a maximum average $\mathrm{Nu}$.

(b)

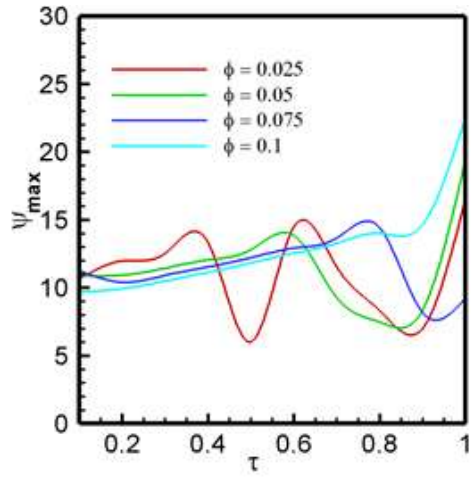

(a)

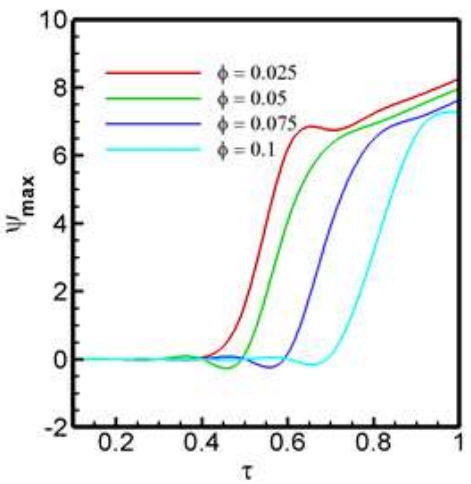

Fig. 10: Effect of the nanoparticle volume fraction on maximum streamfunction value at Ha $=10$ and (a) $\mathrm{Ra}=10^{4}$, (b) $\mathrm{Ra}=10^{5}$

Fig. 10 plots the maximum value of the stream function against dimensionless time. For $R a$ $=10^{4}$, similar to the average $N u$, a jump in maximum stream function is visible. For lower solid volume fractions, the jump occurs at lower values of $\tau$. At $\tau=1$, the lower solid volume fractions show a maximum value for the stream function. With higher fluid momentum, at higher solid volume fractions, the stream function values diminish. Hence, the result is quite obvious. If, however the buoyancy force increases $\left(\mathrm{Ra}=10^{5}\right)$, the pattern varies with dimensionless time. For this case, at $\tau=1$, higher solid volume fractions show a maximum value in the stream function.

\section{Effect of Magnetic Field on Heat Transfer}

With the strong magnetic susceptibility of the ferrofluid, the presence of an external magnetic field creates a Lorentz force that suppresses the fluid motion and hence MHD convection. This field thus provides a means to control heat transfer. The Hartmann number represents how strong the applied magnetic field is. 

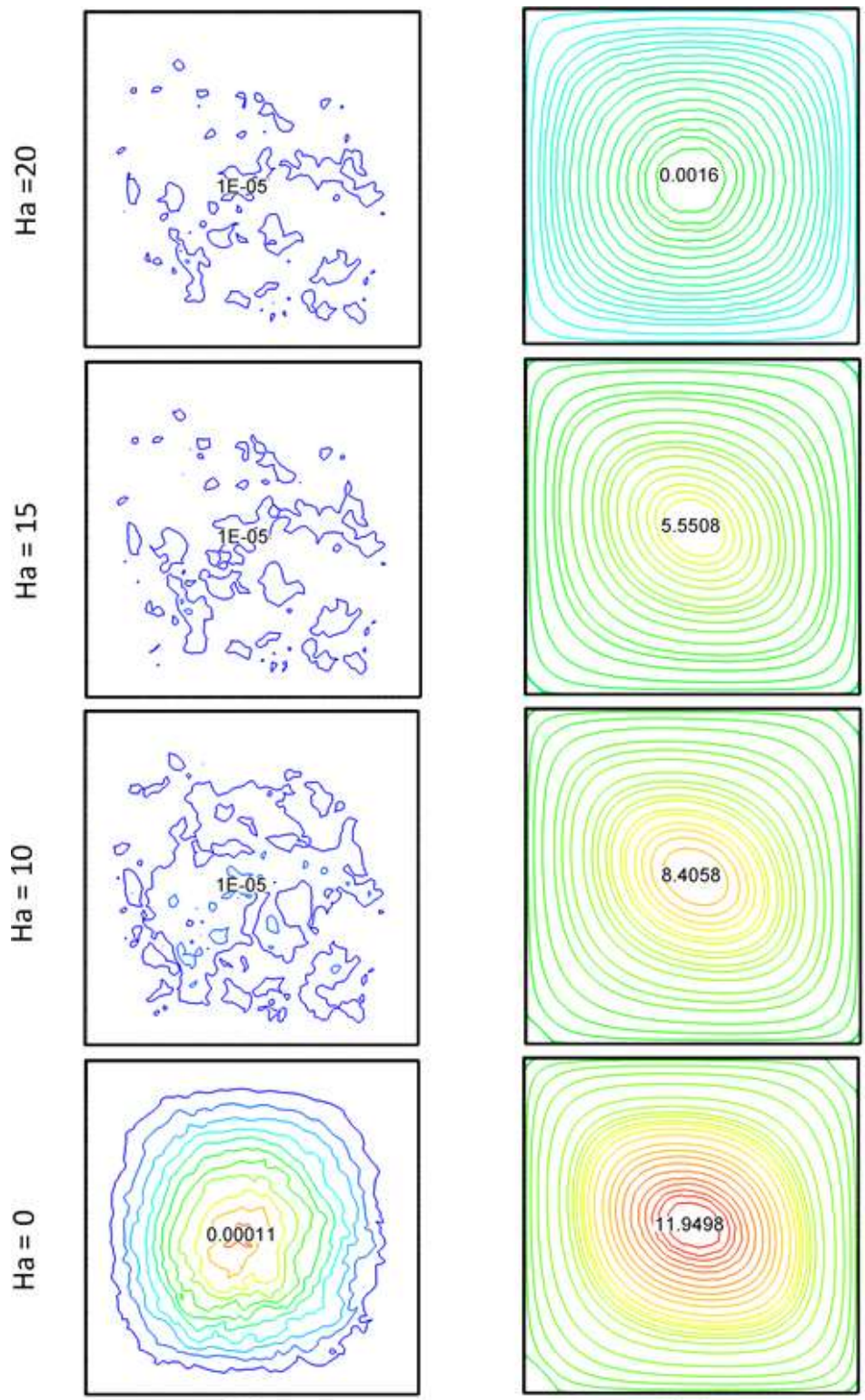

$$
\tau=0.1
$$

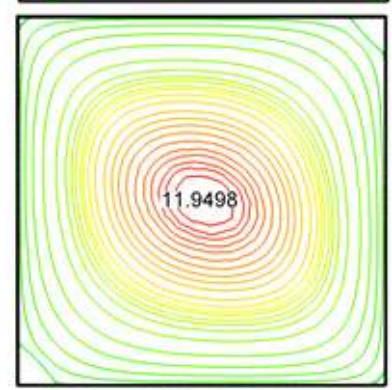

$\tau=1$

Fig. 11: Effect of Hartmann number on streamlines for the selected values of $\tau$ with $\varphi=0.04$ and $\mathrm{Ra}=10^{4}$

In Fig. 11, its effect is shown for $\tau=0.1$ and 1.0 at $R a=10^{4}$. The ferrofluid solid volume fraction is kept constant at $4 \%$. For $\tau=0.1$, no streamline exists for higher $\mathrm{Ha}(=10,15,20)$. At $\mathrm{Ha}=0$, a weak clockwise-rotating vortex is seen. For $\tau=1$, the fluid motion is more stabilized. A clockwiserotating vortex covers the cavity completely. The eye of the vortex is at the center of the cavity. With increasing $\mathrm{Ha}$, the flow strength diminishes as there is a Lorentz force acting against the buoyancy force. For $H a=0$, the value of $\psi_{\max }$ is 11.9498 but decreases to 0.0016 at $\mathrm{Ha}=20$. At higher Rayleigh number $\left(R a=10^{5}\right)$ the buoyancy force increases significantly; hence, streamlines are found for $\tau=0.1$ (Fig. 12). 

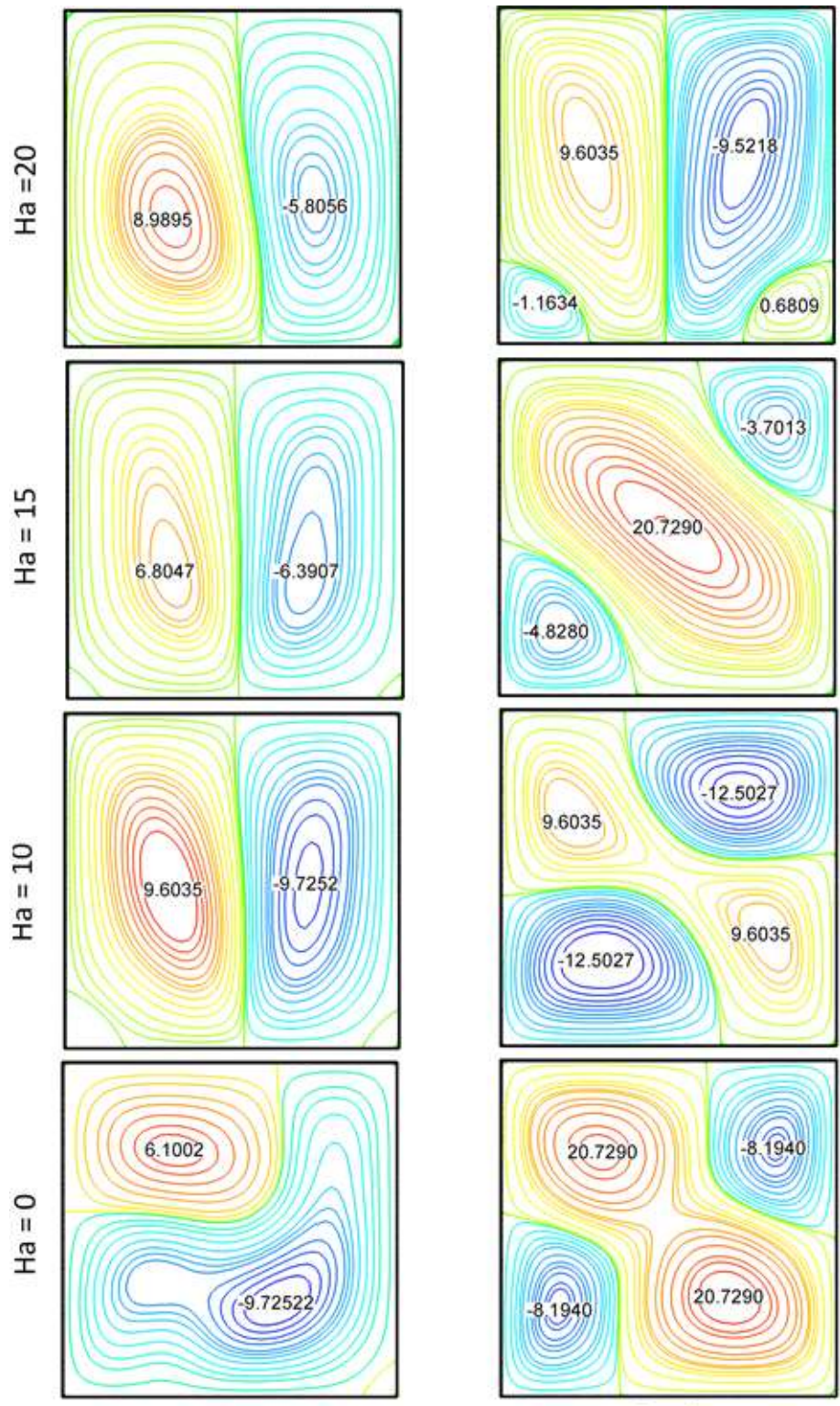

$$
\tau=0.1
$$

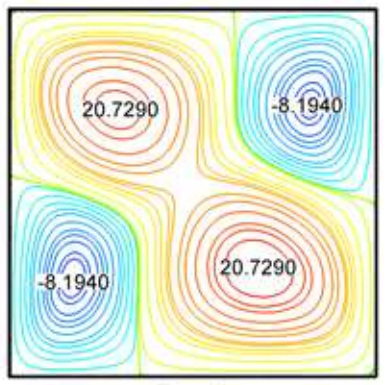

$\tau=1$

Fig. 12: Effect of Hartmann number on streamlines for the selected values of $\tau$ with $\varphi=0.04$ and $\mathrm{Ra}=10^{5}$

At $H a=0$, two vortices are found inside the cavity. A clockwise-rotating vortex is at the topright corner of the cavity along with an anticlockwise-rotating vortex suppressing it. The strength of this anticlockwise-rotating vortex is higher and covers much of the cavity. At $\mathrm{Ha}=$ 10, the buoyancy force effect diminishes and the vortex now becomes symmetric and counteracting. With further increments, $\mathrm{Ha}=15$ and 20, the streamline patterns do not change much and only the strength of the vortices weakens. For $R a=10^{5}$, a significant variation is found at $\tau=1$. For $H a=0$, there are four vortices situated inside the cavity. The strength of the clockwise-rotating vortices is much higher than for the anticlockwise-rotating vortices. At $\mathrm{Ha}$ 
$=10$, the strength of the clockwise-rotating vortices diminishes whereas that for the anticlockwise-rotating vortices increases. Now the anticlockwise-rotating vortices dominate the convection and cover much of the area of the cavity. At $\mathrm{Ha}=15$, the two clockwise-rotating vortex merge and again the strength of vortex is enhanced. Two opposing vortices are also found inside the cavity. At $\mathrm{Ha}=20$, the strength of the vortices diminishes significantly and a general natural convection pattern of streamlines is visible. There are also two minor vortices at the lower-left and lower-right regions of the cavity.
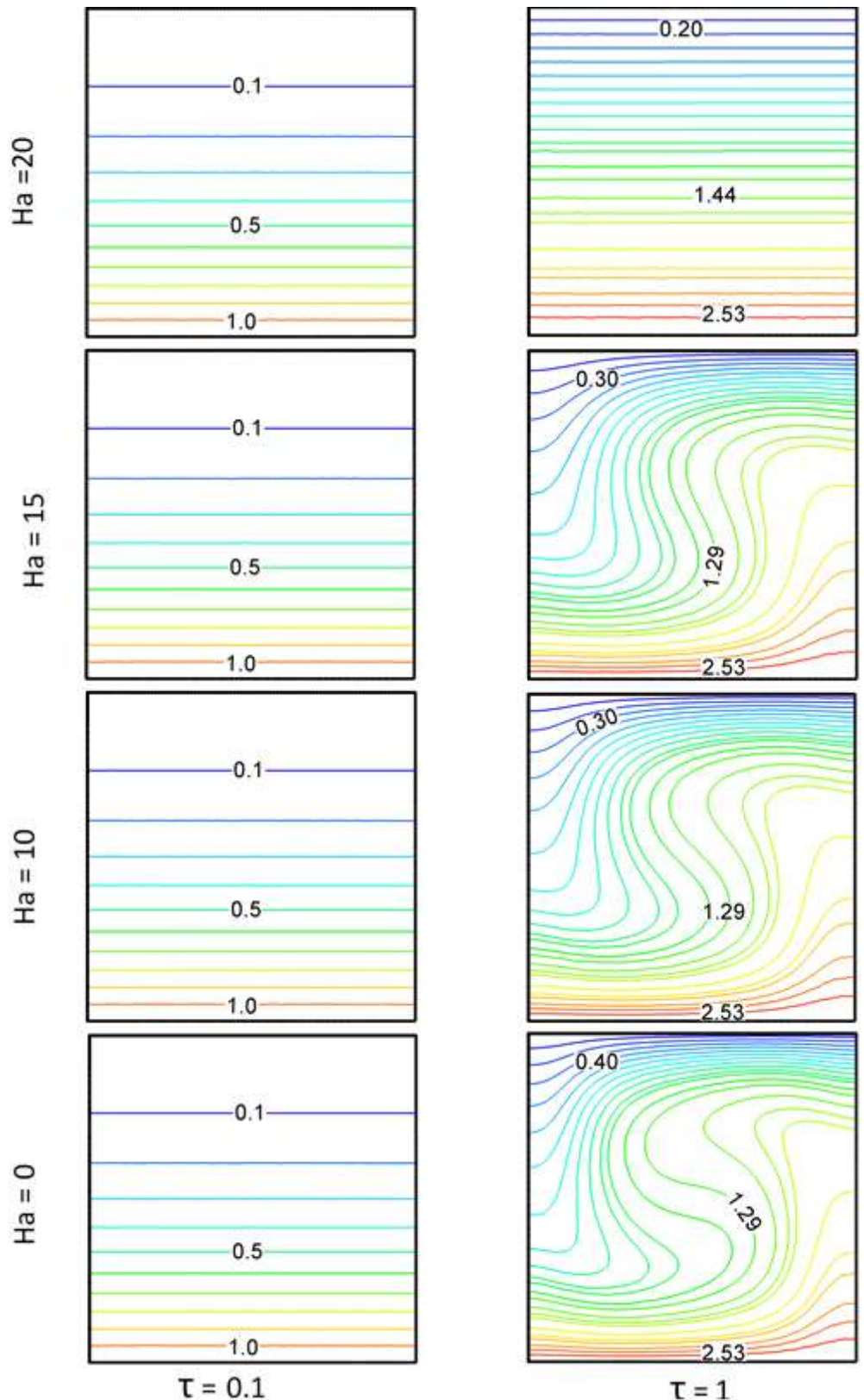

Fig. 13: Effect of Hartmann number on isotherms for the selected values of $\tau$ with $\varphi=0.04$ and $\mathrm{Ra}=10^{4}$ 
Figures 13 and 14 show the effect of Hartmann number for $R a=10^{4}$ and $10^{5}$, respectively, with dimensionless time. For $R a=10^{4}$ and $\tau=0.1$, isotherms are parallel to each other indicating a conduction-dominated heat transfer. Increments in $\mathrm{Ha}$ do not show any variation in isotherms. This phenomenon can be explained by the fact that with smaller time intervals the fluid does not move under the lower buoyancy force available. Therefore, heat transfer occurs between the fluid layers by conduction. At $\tau=1$, when $H a=0$, a significant convection pattern is displayed by the isotherms.
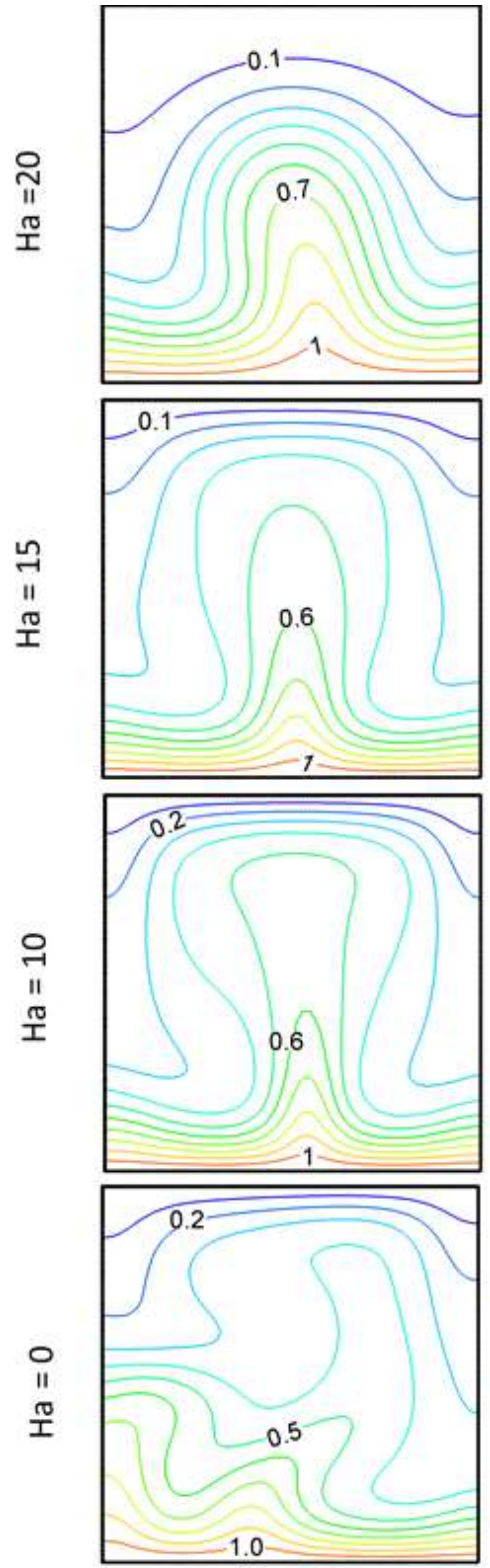

$\tau=0.1$
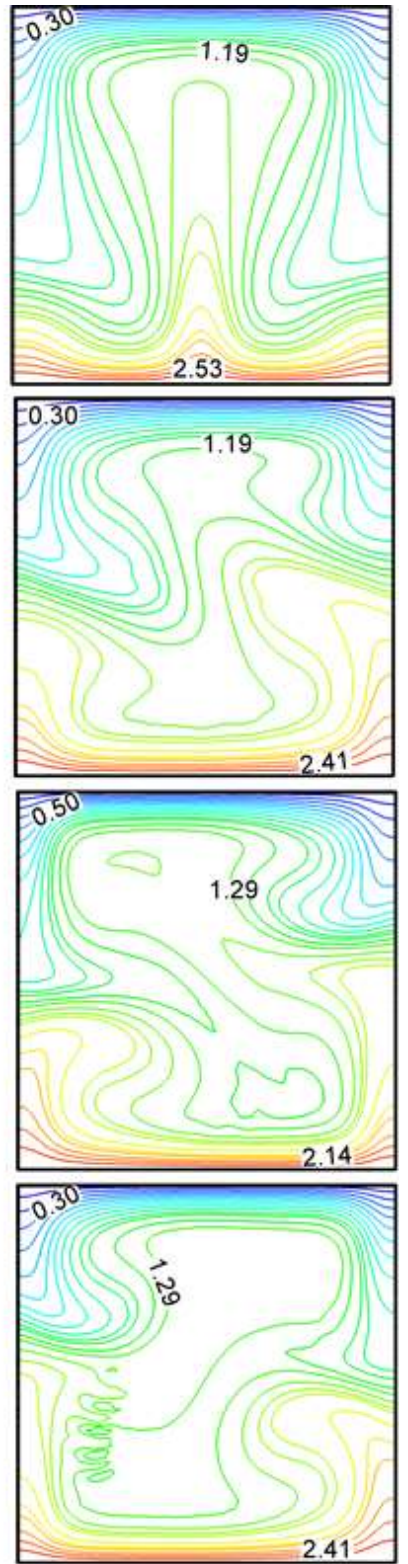

$\tau=1$

Fig. 14: Effect of Hartmann number on isotherms for the selected values of $\tau$ with $\varphi=0.04$ and $\mathrm{Ra}=10^{5}$ 
On incrementing $\mathrm{Ha}$, convection weakens and the heat transfer becomes conduction dominated and the isotherms are less dispersed. At $\mathrm{Ha}=20$, the heat transfer is completely conduction dominated. If $R a$ is increased $\left(\mathrm{Ra}=10^{5}\right)$, a stronger buoyancy force is generated. For $\tau=0.1$ a strong convection pattern is observed. With increments in $H a$, the convectiondominated transfer of heat decreases although a significant convective-dominated transfer of heat remains. At $\tau=1$, strong convection is observed for $\mathrm{Ha}=0$. The convective current is less visible in the center of the cavity. As $\mathrm{Ha}$ increases to 20, the convective heat transfer is weakened and isotherms become comparable with low dimensionless time.

(c)

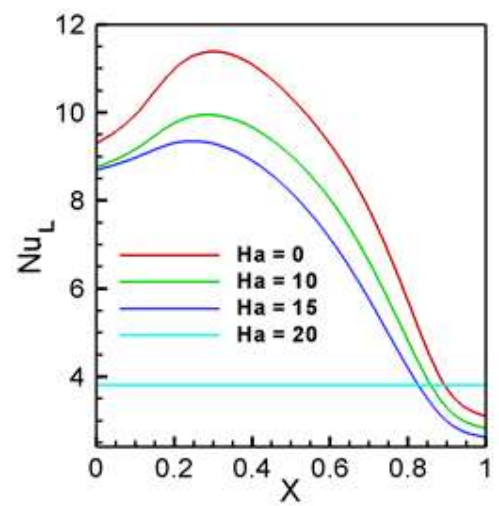

(b)

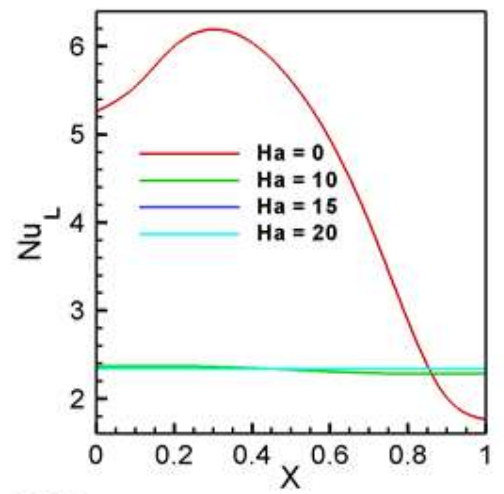

(a)

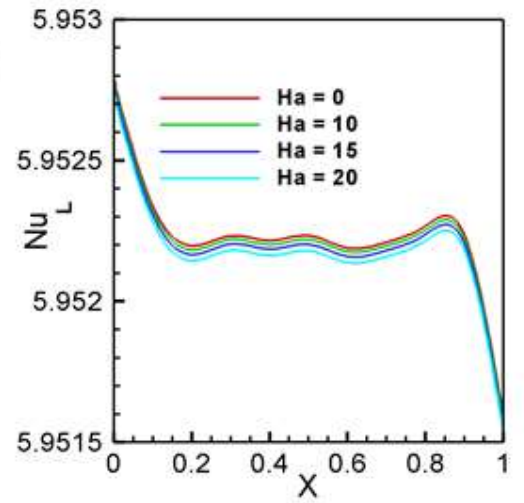

$\mathrm{Ra}=10^{4}$
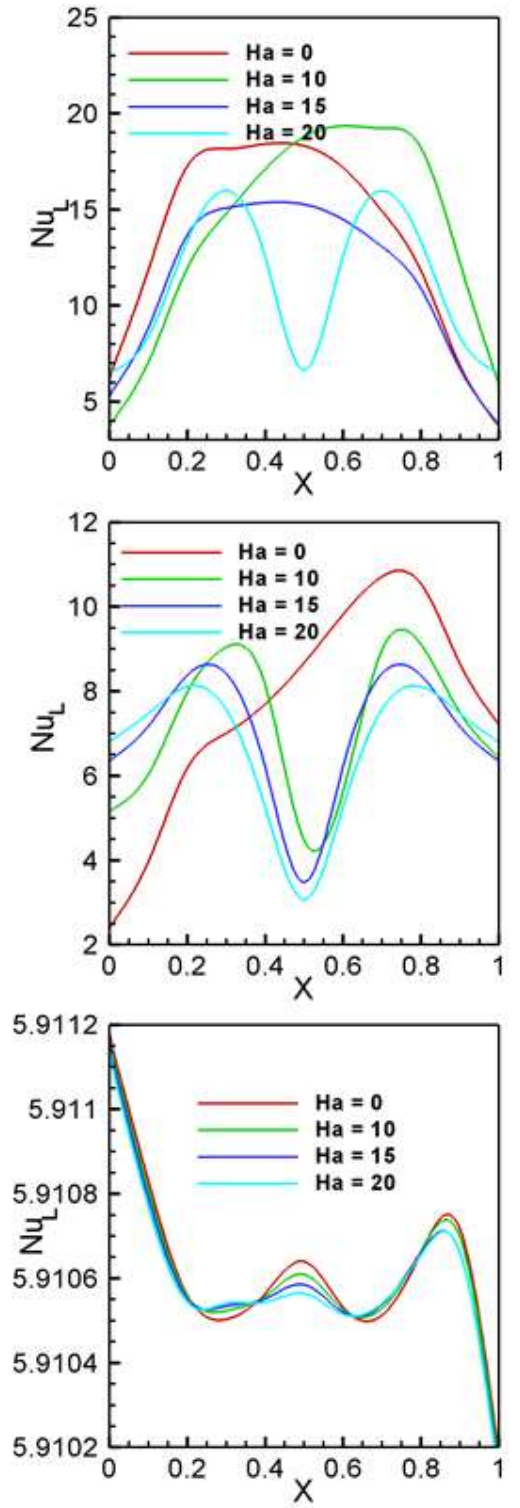

$\mathrm{Ra}=10^{5}$

Fig. 15: Effect of the Hartmann number and dimensionless time on local Nusselt number at $\varphi=0.04$ and (a) $\tau=0.01$, (b) $\tau=0.5$ and (c) $\tau=1$ 
Fig. 15 shows the dependence of $\mathrm{Ha}$ on local $\mathrm{Nu}$ for different dimensionless time. At $\tau=0.01$, local $\mathrm{Nu}$ is almost similar for all $\mathrm{Ha}$. However, lower $\mathrm{Ha}$ implies a comparatively higher local $\mathrm{Nu}$. The local $\mathrm{Nu}$ varies significantly near the boundary region but remain almost constant in the mid-region. For $R a=10^{5}$ and $\tau=0.01$, this mid-region also fluctuates. For $\tau=0.5$ and $R a=10^{4}$, the local $\mathrm{Nu}$ varies significantly when there is no magnetic field present. At higher $\mathrm{Ha}$, local $\mathrm{Nu}$ reduces significantly and becomes constant more or less. For $\mathrm{Ra}=10^{5}$, higher $\mathrm{Ha}$ shows symmetric pattern. For this case $H a=0$ also shows a maximum value for the local $N u$. At $\tau=1$ and $R a=10^{4}$, the $N u$ becomes a maximum near $x=0.3$ and then reduces again significantly for $H a=0-15$. At $H a=20$, the local $N u$ is almost constant and its value is much lower than others. Also, it indicates that at higher $\mathrm{Ha}$, conductive heat transfer is the more dominant. An interesting behavior is observed for $R a=10^{5}$ and $\tau=1$; the local $N u$ is maximum at $H a=10$. This is quite unlikely under other circumstances. When the flow become steady, the effect of the applied magnetic field becomes prominent and generates a higher heat transfer.

(b)

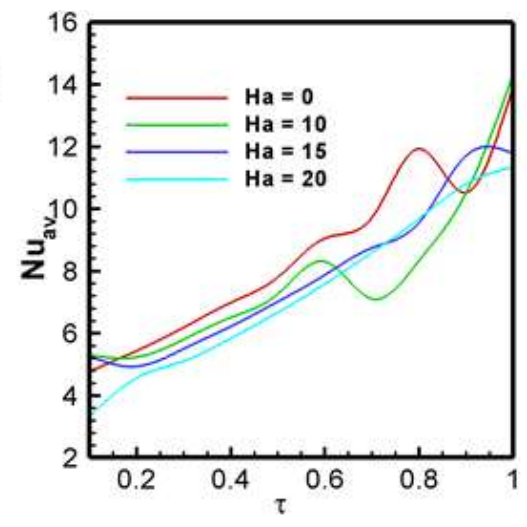

(a)

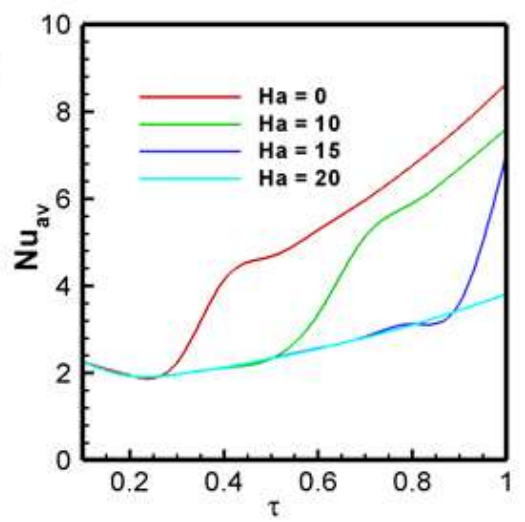

Fig. 16: Effect of the Hartmann number on average Nusselt number at $\varphi=0.04$ and (a) $\mathrm{Ra}=$ $10^{4}$, (b) $\mathrm{Ra}=10^{5}$

Fig. 16 shows the effect of $\mathrm{Ha}$ on the average $N u$. At $R a=10^{4}$, the average $N u$ initially decreases with dimensionless time and then increases $\tau=0.25$. A jump can be observed at a lower value of $\tau$ for $H a=0$. As $H a$ increases, the jump occurs at higher value of $\tau$. Average $N u$ is lower as $H a$ increases for a particular value of $\tau$. For $R a=10^{5}$, an initially higher average $N u$ is found at $H a=0$. Then the value of the average $N u$ decreases compared with that at $H a$ $=0$ up to $\tau=0.85$. After a longer duration $(\tau=1)$, the flow again shows a maximum $N u$ for $H a=10$, and hence a higher heat transfer rate. 
(b)

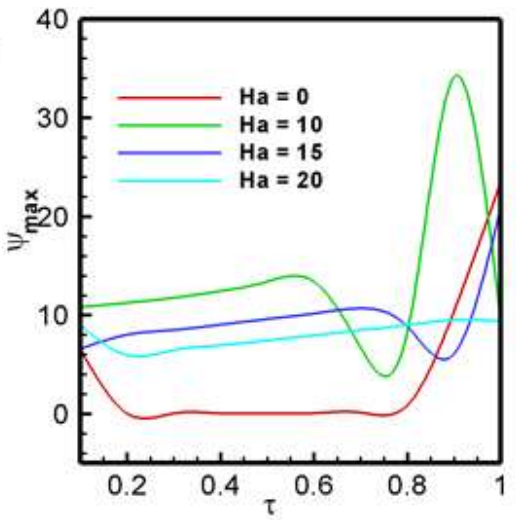

(a)

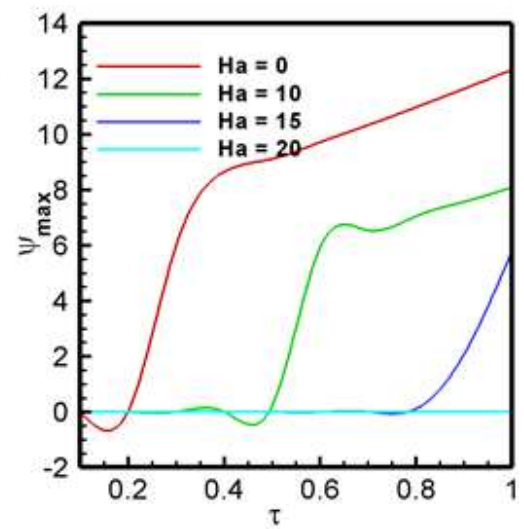

Fig. 17: Effect of the Hartmann number on maximum stream function value at $\varphi=0.04$ and (a) $\mathrm{Ra}=10^{4}$, (b) $\mathrm{Ra}=10^{5}$

Fig. 17 shows the effect of $\mathrm{Ha}$ on the maximum stream function value with dimensionless time. Initially, the stream function value diminishes with time for $R a=10^{4}$, but at a certain value of $\tau$ it experiences a jump. For $H a=0$, this jump occurs at $\tau=0.2$ whereas for $\mathrm{Ha}=10$ and 15 , it occurs at $\tau=0.5$ and 0.8 , respectively. For $H a=20$, no sudden variation in stream function appears. For $R a=10^{4}$, the stream function value diminishes with increments in $H a$. A quite different behavior is observed for $\mathrm{Ra}=10^{5}$. For $\mathrm{Ha}=0$ and 20, the stream function value reduces initially with $\tau$, and then after a certain value of $\tau$, it increases again. For $\mathrm{Ha}=$ 10 and 15, it increases with $\tau$, then decreases suddenly and increases again. The curve for $H a=10$ shows a maximum stream function value for unsteady flow although $H a=0$ shows a maximum stream function value.

The present work has nevertheless some limitations. Here only two-dimensional incompressible flow is considered. In addition, radiation and viscous dissipation are not considered. Also, simple models are used in accounting for thermo physical properties of the ferrofluid. That aside, the present work provides a sufficient explanation of convection and a base for the study of convection with exponential thermal boundary conditions.

\section{Conclusions}

The problem considered is MHD convection with time-dependent exponential boundary condition, which has certain important practical applications. These novel problem specifications were analyzed and the following findings encountered. 
- At lower values of $R a\left(\mathrm{Ra}=10^{4}\right)$, there are no specific streamlines established. After an elapse of time, a flow pattern can be achieved.

- A jump in the average $\mathrm{Nu}$ occurs and a lower solid volume fraction initiates an earlier jump at $R a=10^{4}$.For $R a=10^{5}$, heat transfer is greater for larger solid volume fraction of ferrofluid for unsteady flow. At $\tau=1$, lower solid volume fractions create better heat transfer than at higher solid volume fractions. Hence, the addition of ferromagnetic particles has less effect (suppression of MHD convection) on heat transfer augmentation at higher $R a$.

- Ferrofluids produce a diminishing in stream-function values at lower $R a\left(R a=10^{4}\right)$ but an opposite result is found for higher $R a\left(R a=10^{5}\right)$ at $\tau=1$.

- Incrementing $\mathrm{Ha}$ decreases the heat transfer rate for $\mathrm{Ra}=10^{4}$ with increasing dimensionless time $\tau$ but a maximum heat transfer is found at $H a=10$ for $R a=10^{5}$ at $\tau=1$.

- Lower $\mathrm{Ha}$ values also produce a maximum stream function value at $\tau=1$ for both $\mathrm{Ra}=$ $10^{4}$ and $10^{5}$.

The present investigation lays down a solid foundation for the investigation of time-varying exponential boundary conditions in MHD convection.

\section{REFERENCES}

A.A. Bozhko, G.F. Putin, T. Tynjälä, P. Sarkomaa, Experimental and numerical investigation of wave ferrofluid convection, J. Magn. Magn. Mater. 316 (2007) 433-435. doi:10.1016/j.jmmm.2007.03.105.

A.H. Mahmoudi, I. Pop, M. Shahi, F. Talebi, MHD natural convection and entropy generation in a trapezoidal enclosure using $\mathrm{Cu}$-water nanofluid, Comput. Fluids. 72 (2013) 46-62. doi:10.1016/j.compfluid.2012.11.014.

A.H. Mahmoudi, M. Shahi, A.H. Raouf, A. Ghasemian, Numerical study of natural convection cooling of horizontal heat source mounted in a square cavity filled with nanofluid, Int. Commun. Heat Mass Transf. 37 (2010) 1135-1141. doi:10.1016/j.icheatmasstransfer.2010.06.005.

A.H. Mahmoudi, M. Shahi, A.H. Raouf, A. Ghasemian, Numerical study of natural convection cooling of horizontal heat source mounted in a square cavity filled with nanofluid, Int. Commun. Heat Mass Transf. 37 (2010) 1135-1141.

A.K. Santra, S. Sen, N. Chakraborty, Study of heat transfer augmentation in a differentially heated square cavity using copper-water nanofluid, Int. J. Therm. Sci. 47 (2008) 1113-1122. doi:10.1016/j.ijthermalsci.2007.10.005.

A.V. Belyaev, B.L. Smorodin, The stability of ferrofluid flow in a vertical layer subject to lateral heating and horizontal magnetic field, J. Magn. Magn. Mater. 322 (2010) 2596-2606. doi:10.1016/j.jmmm.2010.03.028.

B.J. Gireesha, B. Mahanthesh, I.S. Shivakumara, K.M. Eshwarappa, Melting heat transfer in boundary layer stagnation-point flow of nanofluid toward a stretching sheet with induced magnetic field, Engineering Science and Technology, an International Journal 19 (2016) 313-321

Bouchmel Mliki, Mohamed Ammar Abbassi, Kamel Guedri, Ahmed Omri, Lattice Boltzmann simulation of natural convection in an L-shaped enclosure in the presence of nanofluid, Engineering Science and Technology, an International Journal 18 (2015) 503-511.

C.J. Ho, M.W. Chen, Z.W. Li, Numerical simulation of natural convection of nanofluid in a square enclosure: Effects due to uncertainties of viscosity and thermal conductivity, Int. J. Heat Mass Transf. 51 (2008) 4506-4516. doi:10.1016/j.ijheatmasstransfer.2007.12.019.

E. Abu-Nada, H.F. Oztop, Effects of inclination angle on natural convection in enclosures filled with $\mathrm{Cu}-$ water nanofluid, Int. J. Heat Fluid Flow. 30 (2009) 669-678.

E. Abu-Nada, H.F. Oztop, Effects of inclination angle on natural convection in enclosures filled with $\mathrm{Cu}$-water nanofluid, Int. J. Heat Fluid Flow. 30 (2009) 669-678. doi:10.1016/j.ijheatfluidflow.2009.02.001. 
F. Selimefendigil, H.F. Öztop, Forced convection of ferrofluids in a vented cavity with a rotating cylinder, Int. J. Therm. Sci. 86 (2014) 258-275. doi:10.1016/j.ijthermalsci.2014.07.007.

F. Selimefendigil, H.F. Öztop, K. Al-Salem, Natural convection of ferrofluids in partially heated square enclosures, J. Magn. Magn. Mater. 372 (2014) 122-133. doi:10.1016/j.jmmm.2014.07.058.

F. Wu, W. Zhou, X. Ma, Natural convection in a porous rectangular enclosure with sinusoidal temperature distributions on both side walls using a thermal non-equilibrium model, Int. J. Heat Mass Transf. 85 (2015) 756-771. doi:10.1016/j.ijheatmasstransfer.2015.02.039.

G.H.R. Kefayati, Natural convection of ferrofluid in a linearly heated cavity utilizing LBM, J. Mol. Liq. 191 (2014) 1-9. doi:10.1016/j.molliq.2013.11.021.

G.R. Kefayati, Simulation of Ferrofluid Heat Dissipation Effect on Natural Convection at an Inclined Cavity Filled with Kerosene/Cobalt Utilizing the Lattice Boltzmann Method, Numer. Heat Transf. Part Appl. 65 (2014) 509-530. doi:10.1080/10407782.2013.836022.

H. Aminfar, M. Mohammadpourfard, S. Ahangar Zonouzi, Numerical study of the ferrofluid flow and heat transfer through a rectangular duct in the presence of a non-uniform transverse magnetic field, J. Magn. Magn. Mater. 327 (2013) 31-42. doi:10.1016/j.jmmm.2012.09.011.

H.C. Brinkman, The Viscosity of Concentrated Suspensions and Solutions, J. Chem. Phys. 20 (1952) 571-571. doi:10.1063/1.1700493.

I. Mejri, A. Mahmoudi, M.A. Abbassi, A. Omri, Magnetic field effect on entropy generation in a nanofluid-filled enclosure with sinusoidal heating on both side walls, Powder Technol. 266 (2014) 340-353. doi:10.1016/j.powtec.2014.06.054.

I. Mejri, A. Mahmoudi, MHD natural convection in a nanofluid-filled open enclosure with a sinusoidal boundary condition, Chem. Eng. Res. Des. 98 (2015) 1-16. doi:10.1016/j.cherd.2015.03.028.

J.C. Maxwell, A treatise on electricity and magnetism, in: 2nd ed., Cambridge: Oxford University Press, 1904.

K.M. Rabbi, S. Saha, S. Mojumder, M.M. Rahman, R. Saidur, T.A. Ibrahim, Numerical investigation of pure mixed convection in a ferrofluid-filled lid-driven cavity for different heater configurations, Alex. Eng. Journ. (2016). doi:10.1016/j.aej.2015.12.021.

M. Ashouri, B. Ebrahimi, M.B. Shafii, M.H. Saidi, M.S. Saidi, Correlation for Nusselt number in pure magnetic convection ferrofluid flow in a square cavity by a numerical investigation, J. Magn. Magn. Mater. 322 (2010) 3607-3613. doi:10.1016/j.jmmm.2010.05.041.

M. Ghasemian, Z. Najafian Ashrafi, M. Goharkhah, M. Ashjaee, Heat transfer characteristics of Fe3O4 ferrofluid flowing in a mini channel under constant and alternating magnetic fields, J. Magn. Magn. Mater. 381 (2015) 158-167. doi:10.1016/j.jmmm.2014.12.078.

M. Jahanshahi, S.F. Hosseinizadeh, M. Alipanah, A. Dehghani, G.R. Vakilinejad, Numerical simulation of free convection based on experimental measured conductivity in a square cavity using Water/SiO2 nanofluid, Int. Commun. Heat Mass Transf. 37 (2010) 687-694. doi:10.1016/j.icheatmasstransfer.2010.03.010.

M. Sheikholeslami, D.D. Ganji, M.M. Rashidi, Ferrofluid flow and heat transfer in a semi annulus enclosure in the presence of magnetic source considering thermal radiation, J. Taiwan Inst. Chem. Eng. 47 (2015) 6-17. doi:10.1016/j.jtice.2014.09.026.

M. Sheikholeslami, M. Gorji-Bandpy, D.D. Ganji, Lattice Boltzmann method for MHD natural convection heat transfer using nanofluid, Powder Technol. 254 (2014) 82-93. doi:10.1016/j.powtec.2013.12.054.

M. Sheikholeslami, M. Gorji-Bandpy, Free convection of ferrofluid in a cavity heated from below in the presence of an external magnetic field, Powder Technol. 256 (2014) 490-498. doi:10.1016/j.powtec.2014.01.079. 
M.A. Mansour, Sameh E. Ahmed, A numerical study on natural convection in porous media-filled an inclined triangular enclosure with heat sources using nanofluid in the presence of heat generation effect, Engineering Science and Technology, an International Journal 18 (2015) 485-495.

M.A.Y. Bakier, Flow in open C-shaped cavities: How far does the change in boundaries affect nanofluid?, Engineering Science and Technology, an International Journal 17 (2014) 116-130.

M.M. Rahman, H.F. Öztop, M. Steele, A.G. Naim, K. Al-Salem, T.A. Ibrahim, Unsteady natural convection and statistical analysis in a CNT-water filled cavity with non-isothermal heating, Int. Commun. Heat Mass Transf. (n.d.). doi:10.1016/j.icheatmasstransfer.2015.02.012.

M.M. Rahman, S. Mojumder, S. Saha, S. Mekhilef, R. Saidur, Augmentation of natural convection heat transfer in triangular shape solar collector by utilizing water based nanofluids having a corrugated bottom wall, Int. Commun. Heat Mass Transf. 50 (2014) 117-127.

M.M. Rahman, S. Mojumder, S. Saha, S. Mekhilef, R. Saidur, Effect of solid volume fraction and tilt angle in a quarter circular solar thermal collectors filled with CNT-water nanofluid, Int. Commun. Heat Mass Transf. 57 (2014) 79-90.

M.M. Rahman, S. Saha, S. Mojumder, A.G. Naim, R. Saidur, T.A. Ibrahim, Effect of sine-squared thermal boundary condition on augmentation of heat transfer in a triangular solar collector filled with different nanofluids, Numer. Heat Transf. Part B Fundam. 68 (2015) 53-74.

M.R. Krishnamurthy, B.C. Prasannakumara, B.J. Gireesha, Rama Subba Reddy Gorla, Effect of chemical reaction on MHD boundary layer flow and melting heat transfer of Williamson nanofluid in porous medium, Engineering Science and Technology, an International Journal 19 (2016) 53-61.

N. Sandeep, C. Sulochana, B. Rushi Kumar, Unsteady MHD radiative flow and heat transfer of a dusty nanofluid over an exponentially stretching surface, Engineering Science and Technology, an International Journal 19 (2016) 227-240.

P. Valinataj-Bahnemiri, A. Ramiar, S.A. Manavi, A. Mozaffari, Heat transfer optimization of two phase modeling of nanofluid in a sinusoidal wavy channel using Artificial Bee Colony technique, Engineering Science and Technology, an International Journal 18 (2015) 727-737.

S. Das, R.N. Jana, O.D. Makinde, Mixed convective magnetohydrodynamic flow in a vertical channel filled with nanofluids, Engineering Science and Technology, an International Journal 18 (2015) 244-255.

S. Eiamsa-ard, K. Kiatkittipong, W. Jedsadaratanachai, Heat transfer enhancement of $\mathrm{TiO} 2 /$ water nanofluid in a heat exchanger tube equipped with overlapped dual twisted-tapes, Engineering Science and Technology, an International Journal 18 (2015) 336-350.

S. Sivasankaran, K.L. Pan, Natural convection of nanofluids in a cavity with Nonuniform Temperature Distributions on Side Walls, Numer. Heat Transf. Part Appl. 65 (2014) 247-268.

S. Sivasankaran, K.L. Pan, Natural Convection of Nanofluids in a Cavity with Nonuniform Temperature Distributions on Side Walls, Numer. Heat Transf. Part Appl. 65 (2014) 247-268. doi:10.1080/10407782.2013.825510.

S.-K. Choi, S.-O. Kim, T.-H. Lee, Dohee-Hahn, Computation of the Natural Convection of Nanofluid in a Square Cavity with Homogeneous and Nonhomogeneous Models, Numer. Heat Transf. Part Appl. 65 (2014) 287-301. doi:10.1080/10407782.2013.831695.

S.M. Aminossadati, B. Ghasemi, Natural convection cooling of a localised heat source at the bottom of a nanofluid-filled enclosure, Eur. J. Mech. - BFluids. 28 (2009) 630-640.

S.M. Aminossadati, B. Ghasemi, Natural convection cooling of a localised heat source at the bottom of a nanofluid-filled enclosure, Eur. J. Mech. - BFluids. 28 (2009) 630-640. doi:10.1016/j.euromechflu.2009.05.006.

S.M. Snyder, T. Cader, B.A. Finlayson, Finite element model of magnetoconvection of a ferrofluid, J. Magn. Magn. Mater. 262 (2003) 269-279. doi:10.1016/S0304-8853(02)01502-0. 
Sameh E. Ahmed, M.A. Mansour, Ahmed Kadhim Hussein, S. Sivasankaran, Mixed convection from a discrete heat source in enclosures with two adjacent moving walls and filled with micropolar nanofluids, Engineering Science and Technology, an International Journal 19 (2016) 364-376.

T-C. Jue, Analysis of combined thermal and magnetic convection ferrofluid flow in a cavity, Int. Commun. Heat Mass Transf. 33 (2006) 846-852. doi:10.1016/j.icheatmasstransfer.2006.02.001.

W.N. Zhou, Y.Y. Yan, Numerical Investigation of the Effects of a Magnetic Field on Nanofluid Flow and Heat Transfer by the Lattice Boltzmann Method, Numer. Heat Transf. Part Appl. 68 (2015) 116. doi:10.1080/10407782.2014.965017.

$--\mathbf{0 - -}$

\section{NOMENCLATURE}

\begin{tabular}{|c|c|c|c|}
\hline$B_{0}$ & magnetic induction $\left(\mu_{0} H\right)$ & $\beta$ & thermal expansion coefficient $\left(\mathrm{K}^{-1}\right)$ \\
\hline$c_{p}$ & specific heat $\left(\mathrm{J} \mathrm{kg}^{-1} \mathrm{k}^{-1}\right)$ & $\delta$ & dependent non-dimensional variables \\
\hline$g$ & gravitational acceleration $\left(\mathrm{ms}^{-2}\right)$ & $\Gamma_{\delta}$ & diffusion term in Eq. (1) \\
\hline$H$ & enclosure height $(\mathrm{m})$ & $\phi$ & solid volume fraction \\
\hline $\mathrm{Ha}$ & Hartmann number & $\mu$ & dynamic viscosity $\left(\mathrm{kg} \mathrm{m}^{-1} \mathrm{~s}^{-1}\right)$ \\
\hline K & thermal conductivity $\left(\mathrm{Wm}^{-1} \mathrm{k}^{-1}\right)$ & $v$ & kinematic viscosity $\left(\mathrm{m}^{2} \mathrm{~s}^{-1}\right)$ \\
\hline$L$ & length of the enclosure (m) & $\tau$ & dimensionless time \\
\hline$N u$ & Nusselt number & $\Theta$ & non-dimensional temperature \\
\hline$p$ & dimensional pressure $\left(\mathrm{kg} \mathrm{m}^{-1} \mathrm{~s}^{-2}\right)$ & $\rho$ & density $\left(\mathrm{kg} \mathrm{m}^{-3}\right)$ \\
\hline$P$ & dimensionless pressure & $\sigma$ & electrical conductivity \\
\hline $\operatorname{Pr}$ & Prandtl number & $\psi$ & streamfunction \\
\hline$R a$ & Rayleigh number & & \\
\hline $\mathrm{S}_{\delta}$ & source term in Eq. (1) & & \\
\hline$T$ & fluid temperature $(\mathrm{K})$ & \multicolumn{2}{|c|}{ Subscripts } \\
\hline$t$ & elapsed time (s) & $a v$ & average \\
\hline$U$ & $\begin{array}{l}\text { horizontal velocity component } \\
\left(\mathrm{ms}^{-1}\right)\end{array}$ & $h$ & heat source \\
\hline$U$ & $\begin{array}{l}\text { dimensionless horizontal velocity } \\
\text { component }\end{array}$ & $c$ & cold \\
\hline$V$ & vertical velocity component $\left(\mathrm{ms}^{-1}\right)$ & $\mathrm{f}$ & fluid \\
\hline$V$ & $\begin{array}{l}\text { dimensionless vertical velocity } \\
\text { component }\end{array}$ & $\mathrm{ff}$ & ferrofluid \\
\hline$x$ & horizontal coordinate $(\mathrm{m})$ & $\mathrm{s}$ & ferroparticle \\
\hline$X$ & $\begin{array}{l}\text { dimensionless horizontal } \\
\text { coordinate }\end{array}$ & $\max$ & maximum \\
\hline$y$ & vertical coordinate $(\mathrm{m})$ & $\min$ & minimum \\
\hline$Y$ & dimensionless vertical coordinate & $\mathrm{L}$ & local \\
\hline \multicolumn{4}{|c|}{ Greek symbols } \\
\hline$\alpha$ & thermal diffusivity $\left(\mathrm{m}^{2} \mathrm{~s}^{-1}\right)$ & & \\
\hline
\end{tabular}


ISSN: $2409-3629$

Online Archive Link: $\underline{\text { https://abc.us.org/ojs/index.php/ei/issue/archive }}$ 